\title{
MULTILEVEL BALANCING DOMAIN DECOMPOSITION AT EXTREME SCALES *
}

\author{
SANTIAGO BADIA B $^{\ddagger}$, ALBERTO F. MARTÍN ${ }^{\dagger} \ddagger$, AND JAVIER PRINCIPE A $^{\ddagger}$
}

\begin{abstract}
In this paper we present a fully-distributed, communicator-aware, recursive, and interlevel-overlapped message-passing implementation of the multilevel balancing domain decomposition by constraints (MLBDDC) preconditioner. The implementation highly relies on subcommunicators in order to achieve the desired effect of coarse-grain overlapping of computation and communication, and communication and communication among levels in the hierarchy (namely inter-level overlapping). Essentially, the main communicator is split into as many non-overlapping subsets of MPI tasks (i.e., MPI subcommunicators) as levels in the hierarchy. Provided that specialized resources (cores and memory) are devoted to each level, a careful re-scheduling and mapping of all the computations and communications in the algorithm lets a high degree of overlapping to be exploited among levels. All subroutines and associated data structures are expressed recursively, and therefore MLBDDC preconditioners with an arbitrary number of levels can be built while re-using significant and recurrent parts of the codes. This approach leads to excellent weak scalability results as soon as level-1 tasks can mask coarser-levels duties. We provide a model to indicate how to choose the number of levels and coarsening ratios between consecutive levels and determine qualitatively the scalability limits for a given choice. We have carried out a comprehensive weak scalability analysis of the proposed implementation for the 3D Laplacian and linear elasticity problems. Excellent weak scalability results have been obtained up to 458,752 IBM BG/Q cores and 1.8 million MPI tasks, being the first time that exact domain decomposition preconditioners (only based on sparse direct solvers) reach these scales.
\end{abstract}

1. Introduction. The simulation of scientific and engineering problems governed by partial differential equations (PDEs) involves the solution of sparse linear systems. The time spent in an implicit simulation at the linear solver relative to the overall execution time grows with the size of the problem and the number of cores [22]. In order to satisfy the ever increasing demand of reality and complexity in the simulations, scientific computing must advance in the development of numerical algorithms and implementations that will efficiently exploit the largest amounts of computational resources, and a massively parallel linear solver is a key component in this process.

The growth in computational power passes now through increasing the number of cores in a chip, instead of making cores faster. The next generation of supercomputers, able to reach 1 exaflop/s, is expected to reach billions of cores. Thus, the future of scientific computing will be strongly related to the ability to efficiently exploit these extreme core counts [1].

Only numerical algorithms with all their components scalable will efficiently run on extreme scale supercomputers. On extreme core counts, it will be a must to reduce communication and synchronization among cores, and overlap communication with computation. At the largest scales, linear solvers are based on preconditioned Krylov subspace methods. Algorithmically scalable preconditioners include (algebraic) multigrid (MG) [30] and some domain decomposition (DD) algorithms [31]. However, this theoretical property is not enough for practical weak scalability, since the preconditioner itself must allow for a massively scalable implementation. Today's most scalable algorithms/implementations present practical limits of parallelism, e.g., due to the small, coarse problems to be solved in the hierarchical process for DD/AMG, and the loss of sparsity and denser communication patterns at coarser levels of AMG [7].

DD preconditioners make explicit use of the partition of the global mesh, e.g., for the finite element (FE) integration, into sub-meshes (subdomains), and provide a natural framework for the development of fast and robust parallel solvers tailored for distributed-memory machines. One-level DD algorithms involve the solution of local problems and nearest-neighbors communications. A (second level) coarse correction (coupling all subdomains) is required to have algorithmic scalability, but it can also harm the practical (CPU time) weak scalability. Two-level DD algorithms include the Balancing Neumann-

\footnotetext{
${ }^{\dagger}$ Centre Internacional de Mètodes Numèrics a l'Enginyeria (CIMNE), Parc Mediterrani de la Tecnologia, UPC, Esteve Terradas 5, 08860 Castelldefels, Spain (\{sbadia,amartin,principe\}@cimne.upc.edu).

${ }^{\ddagger}$ Universitat Politècnica de Catalunya, Jordi Girona 1-3, Edifici C1, 08034 Barcelona, Spain.

* This work has been funded by the European Research Council under the FP7 Programme Ideas through the Starting Grant No. 258443 - COMFUS: Computational Methods for Fusion Technology and the FP7 NUMEXAS project under grant agreement 611636. A. F. Martín was also partially funded by the Generalitat de Catalunya under the program "Ajuts per a la incorporació, amb caràcter temporal, de personal investigador júnior a les universitats públiques del sistema universitari català PDJ 2013'. We acknowledge PRACE for awarding us access to FERMI based in Bologna (Italy) at CINECA, and GCS to JUQUEEN based on Jülich (Germany) at JSC. We gratefully acknowledge JSC staff in general, and Dirk Broemmel in particular, for their support in porting/debugging FEMPAR and its dependencies to/on JUQUEEN.
} 
Neumann preconditioner (BNN) [23], the Balancing DD by Constraints preconditioner (BDDC) [13], and FETI-DP preconditioners [15]. The practical scalability limits of a two-level DD implementation is determined by the coarse solver computation, whose size increases (at best) linearly with respect to the number of subdomains. The coarse problem rapidly becomes the bottleneck of the algorithm as we increase the number of cores, since it irremediably produces a severe parallel efficiency loss (all the cores not involved in the coarse solver computation are idling).

Weak scalability can be sustained even when incurring in notable parallel efficiency loss, by increasing the number of cores dealing with the coarse solver, e.g., by means of a message-passing sparse direct solver as the one in MUMPS [2]. The complexity of this type of solvers is quadratic for three dimensional problems, and a quadratic increase of the number of cores is needed to keep constant the computational time. However, the scalability of sparse direct solvers is limited to some hundreds of cores, harming the overall weak scalability of the two-level BDDC method at some point [3].

A salient feature of BDDC methods is the fact that the constrained Neumann and Dirichlet local problems, as well as the coarse problem, can be computed in an inexact way, e.g., using one AMG cycle without affecting the algorithmic scalability of the method [14]. The use of inexact coarse solvers is also possible for FETI-DP methods, after some modifications [20]. Since AMG solvers maintain weak scalability much further than message-passing sparse direct methods, inexact versions of BDDC and FETI-DP methods exhibit improved weak scalability, even though such implementations still incur in the parallel efficiency loss commented above. Inexact FETI-DP methods, in which local problems are computed with direct solvers and the coarse problem is approximated using AMG, have been exploited in $[20]$.
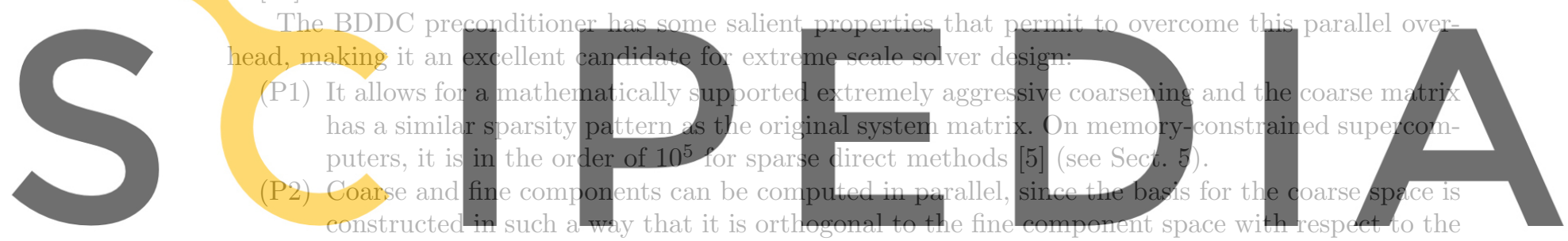
inner product endowed by the system matrix [5]

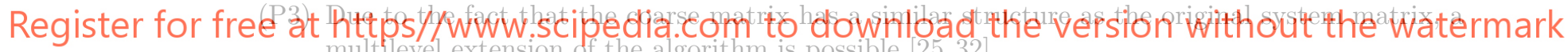
multilevel extension of the algorithm is possible 25,32].

Property (P1) is readily exploited in any BDDC implementation.

The efficient exploitation of (P2), i.e., the orthogonality between coarse and fine spaces, is not trivial. However, this property makes possible a parallel computation of coarse and fine corrections, i.e., overlapped in time. In [5], we have classified all the duties in an exact (i.e., using sparse direct solvers) BDDC-PCG algorithm into fine and coarse duties. These duties have been re-scheduled to achieve the maximum degree of overlapping while preserving data dependencies. The actual implementation of this idea requires significant code refactoring, since it involves a switch from SPMD (Single Program Multiple Data) to a MPMD (Multiple Program Multiple Data) parallel execution mode; cores are divided into those having fine grid duties and those having coarse grid duties. This bulk-asynchronous approach reduces synchronization among cores, and overlaps communications/computations, following the exascale solver paradigm [1]. It has been exploited in [5], where we have performed scalability analyses for the 3D Poisson and linear elasticity problems on a pair of state-of-the-art multicore-based distributed-memory machines (HELIOS and CURIE). Excellent weak scalability has been attained up to $27 \mathrm{~K}$ cores for reasonably high local problem sizes; both local and coarse problems were solved by using the multi-threaded sparse direct solver PARDISO [28]. Further, the clear reduction of computing time and memory requirements of inexact solvers compared to sparse direct ones made possible to get in [6] excellent weak scalability results for the inexact/overlapped implementation of the two-level BDDC preconditioner, up to 93,312 cores and 20 billion unknowns on JUQUEEN.

With regard to (P3), a multilevel BDDC (MLBDDC) algorithm has been proposed in [25], where the coarse problem at the next BDDC level is approximated by its BDDC approximation. An implementation of the MLBDDC method that does not exploit (P2) can be found in [29]. Even though the CPU cost of the coarse problem is reduced, since the coarse problem is still serialized with respect to the fine component, the implementation in [25] still suffers from parallel efficiency loss. Further, since the condition number 
bound slightly increases with the number of levels [25], the detailed numerical experiments in [29] are not conclusive to prove the benefit of the multilevel extension (see, e.g., Tables 4, 7, and 8 in [29]).

In this work, we extend the approach in [5] for the two-level BDDC method to MLBDDC. Exact two-level methods with serial coarse solver are effective till some tens of thousands of cores. Beyond that point, the coarse problem cannot be masked anymore by fine duties. In order to go to larger core counts, we extend here the approach in [5] for the two-level BDDC method to MLBDDC, i.e., to exploit both (P2) and (P3). We present a fully-distributed, communicator-aware, recursive, and interleveloverlapped message-passing implementation of the MLBDDC preconditioner. Fully-distributed (versus centralized) means that all data structures (and thus associated computations/communications) are distributed among cores, including the coarse-grid problem at all levels of the MLBDDC preconditioning hierarchy. Communicator-awareness refers to the fact that the codes highly rely on subcommunicators in order to achieve the desired effect of coarse-grain overlapping of computation and communication, and communication and communication among levels in the hierarchy (namely inter-level overlapping). Essentially, the main communicator is split into as many non-overlapping subsets of MPI tasks (i.e.. MPI subcommunicators) as levels in the hierarchy. On a given intermediate $k$-th level, the coarse-grid problem built on level $k-1$ is distributed among the subset of MPI tasks devoted to level $k$. Provided that specialized hardware resources (cores and memory) are devoted to each level, a careful re-scheduling and mapping of all the computations and communications in the algorithm lets a high degree of overlapping to be exploited among levels. Finally, recursive implementation means that all subroutines and associated data structures are expressed recursively, and therefore MLBDDC preconditioners with an arbitrary number of levels can be built while re-using recursively recurrent parts of the code (e.g., communicator

creation, local solvers, inter-level data transfers, etc.

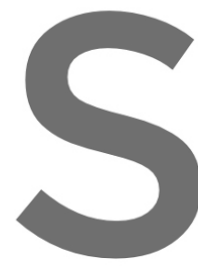

levels duties. We provi between consecutive le we present a compreh dimensional (3D) Lapl: obtained up to 458,752
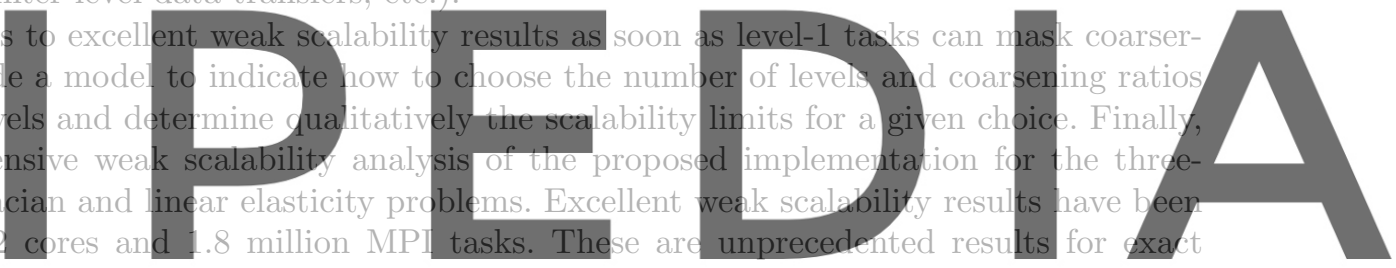

domain decomposition preconditioners (equipped with sparse direct solvers) that represent more than Register one order of magnitude (in terms of scalability limits) improvement with respect to the best results

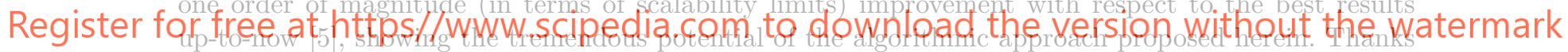

to these results, the software platform FEMPAR [4] in which we have implemented the MLBDDC algorithm is in the High-Q club (since 2014) of the most scalable codes on the JUQUEEN IBM Blue Gene/Q supercomputer [11].

Let us summarize the contributions of this work:

- Novel recursive implementation of MLBDDC methods based on multilevel overlapping strategies in time, in order to fully mask the coarse-grained tasks on much larger core counts than two-level methods.

- Detailed exposition about how to efficiently exploit this novel approach on state-of-the-art supercomputers, including the exploitation of recursion in a multilevel setting for the overlapped deployment of tasks and the construction of sub-communicators.

- Comprehensive weak scalability analysis of the proposed strategy for both Laplacian and linear elasticity problems on the IBM Blue Gene/Q supercomputer, up to 458,752 cores and 1.8 million MPI tasks (subdomains).

This work is structured as follows. The MLBDDC preconditioner is presented in Sect. 2. In Sect. 3, we present an overlapped MLBDDC implementation of the algorithm, which overlaps multiple level computations, and we elaborate a model to determine the scalability limits for a given choice of the number of levels and the coarsening ratios between consecutive levels. In Sect. 4 we provide some implementation keys such as the recursive creation of MPI sub-communicators. In Sect. 5, we report a comprehensive set of numerical experiments. Finally, in Sect. 6, we draw some conclusions and define future lines of work.

2. Multilevel balancing domain decomposition. In this section we state the MLBDDC preconditioner. In Sect. 2.1, we introduce some basic notation. Sect. 2.2 is devoted to the two-level BDDC algorithm. Finally, the MLBDDC algorithm is defined in a recursive way in Sect. 2.3, relying on the con- 
cepts introduced in Sect. 2.2 for the two-level algorithm. In any case, the description of the algorithms is concise, and we refer the reader to $[10,24]$ for a detailed exposition of two-level BDDC methods, and to [25] for the multilevel extension. Further, a thorough presentation of practical implementation details for domain decomposition methods can be found in [4].

2.1. Problem setting. Let us consider a bounded polyhedral domain $\Omega \subset \mathbb{R}^{d}$ with $d=2,3$ and a quasi-uniform partition (mesh) $\mathcal{T}_{0}$ with characteristic size $h_{0}$. Usually, $\mathcal{T}_{0}$ is a partition into tetrahedra/hexahedra for $d=3$ or triangles/quadrilaterals for $d=2$. We consider a quasi-uniform partition $\mathcal{T}_{1}$ of $\mathcal{T}_{0}$ into $n_{1}^{\text {sbd }}$ sub-meshes, which induces a non-overlapping domain decomposition of $\Omega$ into subdomains $\Omega_{1}^{i}, i=1, \ldots, n_{1}^{\text {sbd }}$ (of characteristic size $h_{1}$ ). The interface of $\Omega_{1}^{i}$ is defined as $\Gamma_{1}^{i}:=\partial \Omega_{1}^{i} \backslash \partial \Omega$ and the whole interface (skeleton) of the domain decomposition is $\Gamma_{1}:=\bigcup_{i=1}^{n_{1}^{\mathrm{sbd}}} \Gamma_{1}^{i}$.

As model problem, we study the Poisson problem and linear elasticity on $\Omega$, for an arbitrary forcing term and boundary conditions (as soon as the problem is well-posed). Let us consider a conforming FE space $\overline{\mathbb{V}}_{1} \subset H^{1}(\Omega)$. We denote by $\mathbb{V}_{1}^{i}$ the restriction of $\overline{\mathbb{V}}_{1}$ into $\Omega_{1}^{i} \in \mathcal{T}_{1}$, i.e., local FE spaces.

$\mathbb{V}_{1}:=\mathbb{V}_{1}^{1} \times \ldots \times \mathbb{V}_{1}^{n_{1}^{\text {sbd }}}$ is the global $\mathrm{FE}$ space of functions that can be discontinuous on $\Gamma_{1}$. Let us also define the projection $E_{1}: \mathbb{V}_{1} \rightarrow \overline{\mathbb{V}}_{1}$ as some weighted average of interface values (see, e.g., [24]). We note that $\mathcal{T}_{0}$ and the FE type defines $\overline{\mathbb{V}}_{1}$, whereas $\mathcal{T}_{1}$ is also required to define the local and discontinuous spaces $\mathbb{V}_{1}^{i}$ and $\mathbb{V}_{1}$, respectively. The Galerkin approximation of the problem at hand (with respect to $\bar{V}_{1}$ ) leads to the global linear system of equations to be solved:

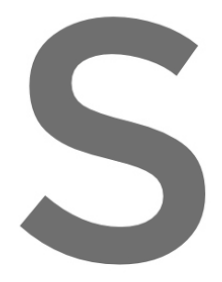

$$
A_{1} x_{1}=f_{1} .
$$

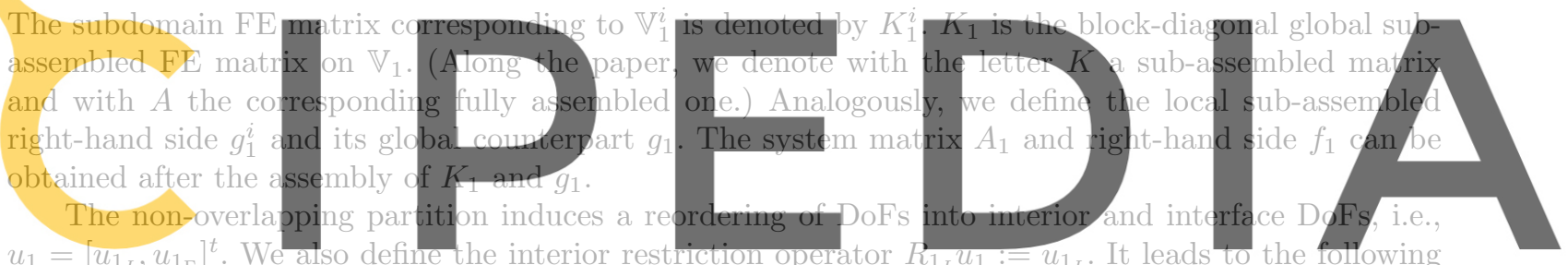

Register for free at https//www.scipedia.com to download the verssion without the watermark

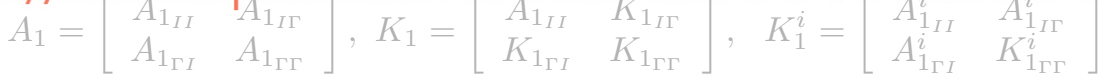

respectively. Matrices $A_{1_{I I}}, A_{1_{I \Gamma}}, A_{1_{\Gamma I}}$ and $K_{1_{\Gamma \Gamma}}$ present a block-diagonal structure (very amenable to parallelization). Matrices $K_{1_{I \Gamma}}$ and $K_{1_{\Gamma I}}$ are trivial extensions (by zeroes) of $A_{1_{I \Gamma}}$ and $A_{1_{\Gamma I}}$, respectively.

2.2. Two-level BDDC preconditioner. In the sequel, we state the two-level BDDC algorithm, describing the set-up of the preconditioner and its application. The input required to set-up the BDDC preconditioner is $\left(\mathcal{T}_{1}, \overline{\mathbb{V}}_{1}, K_{1}\right)$. We recall that $\mathcal{T}_{1}$ is a subdomain partition, $\overline{\mathbb{V}}_{1}$ is the global FE space, and $K_{1}$ is the global sub-assembled matrix.

The construction of the BDDC preconditioner requires a partition of the degrees of freedom (DoFs) corresponding to $\overline{\mathbb{V}}_{1}$ on $\Gamma_{1}$ into objects, which can be corners, edges, or faces. Next, we associate to some (or all) of these objects a coarse DoF. The coarse DoFs can be the values of the function at the corners, or the mean values of the function on edges/faces. Three common variants of the BDDC method are referred as $\mathrm{BDDC}(\mathrm{c}), \mathrm{BDDC}(\mathrm{ce})$ and $\mathrm{BDDC}(\mathrm{cef})$, where we enforce continuity on only corner coarse DoFs, corner and edge coarse DoFs, and corner, edge, and face coarse DoFs, respectively. The definition of the objects and the coarse DoFs can be implemented as an automatic process for arbitrary partitions and physical problems (see [4]). It involves the use of a kernel detection mechanism in order to preserve the well-posedness of the BDDC preconditioner (see [33]). Once we have defined the coarse DoFs, we can define the BDDC FE space $\widetilde{\mathbb{V}}_{1}$ as the subspace of functions in $\mathbb{V}_{1}$ that are continuous on coarse DoFs; clearly, $\overline{\mathbb{V}}_{1} \subset \tilde{\mathbb{V}}_{1} \subset \mathbb{V}_{1}$.

The BDDC preconditioner is a Schwarz-type preconditioner that combines interior corrections with corrections in the BDDC space $\tilde{\mathbb{V}}_{1}$ (see, e.g., $[10,31]$ ). We define the interior correction operator as $P_{1}:=R_{1_{I}}^{t} A_{1_{I I}}^{-1} R_{1_{I}}$, which involves the set-up of $\left(A_{1_{I I}}^{i}\right)^{-1}$, i.e., local Dirichlet problems. The BDDC correction is expressed as $E_{1} \tilde{K}_{1}^{-1} E_{1}^{t}$, where $\tilde{K}_{1}$ is the Galerkin projection of $K_{1}$ onto $\tilde{\mathbb{V}}_{1}$. 
The set-up of the BDDC correction requires some elaboration. Let us consider a decomposition of the BDDC space $\tilde{\mathbb{V}}_{1}$ into a fine space $\tilde{\mathbb{V}}_{1_{f}}$ of vectors that vanish on coarse DoFs and the $K$-orthogonal complement $\tilde{\mathbb{V}}_{1_{c}}$, denoted as the coarse space. As a result, the BDDC FE problem can be decomposed into fine and coarse components, i.e., $\tilde{x}_{1}=\tilde{K}_{1}^{-1} E_{1}^{t} r_{1}=x_{1_{f}}+x_{1_{c}}$. Since fine and coarse spaces are $K_{1}$-orthogonal by definition, they can be computed in parallel.

The fine space functions in $\tilde{\mathbb{V}}_{1_{f}}$ vanish on coarse DoFs (which are the only DoFs that involve continuity among subdomains). Due to the $K_{1}$-orthogonality, the fine component can be defined as $x_{1_{f}}:=E_{1} K_{1_{f}}^{-1} E_{1}^{t}$, where $K_{1_{f}}$ is the Galerkin projection of $K_{1}$ onto $\tilde{V}_{1_{f}}$. Let us note that, since the coarse DoFs are fixed (to zero) in the fine correction, and these are the only DoFs that couple subdomains, the set-up of $K_{1_{f}}^{-1}$ only involves the solution of local Neumann problems with constrained values at the corresponding subdomain coarse DoFs, denoted by $\left(K_{1_{f}}^{i}\right)^{-1}$. For a comprehensive exposition of the implementation issues regarding the solution of constrained Neumann problems we refer to [4].

The coarse space $\tilde{\mathbb{V}}_{1} \subset \tilde{\mathbb{V}}_{1}$ is built as $\tilde{\mathbb{V}}_{1 c}=\operatorname{span}\left\{\phi_{1}^{1}, \phi_{1}^{2}, \ldots, \phi_{1}^{\text {cts }}\right\}$, where $\phi_{1}^{\alpha} \in \tilde{\mathbb{V}}_{1}$ is the coarse shape function associated to the coarse DoF $\alpha$, i.e., it takes value one on $\alpha$ and vanish on the rest of coarse DoFs. As a result, its computation also involves $K_{1_{f}}^{-1}$ (see [4]). Let us note that the support of $\phi_{1}^{\alpha}$ is the set of subdomains that contain $\alpha$. Thus, at every subdomain we only compute the coarse space basis functions related to owned coarse DoFs. We denote by $\Phi_{1}$ the matrix with columns the coarse shape functions. We define the coarse matrix $K_{1_{c}}$ as the assembly of the subdomain local matrices $K_{1_{c}}^{i}=\Phi_{1}^{i t} K_{1}^{i} \Phi_{1}^{i}$, for $i=1, \ldots, n_{1}^{\text {sbd }}$. Further, in a two-level algorithm, we have to set-up $A_{1_{c}}^{-1}$, e.g. after assembling the subdomain contributions in one processor. Using the fact that $P_{1} A_{1} P_{1}=P_{1}$, the two-level BDDC preconditioner can be stated in a compact form as:
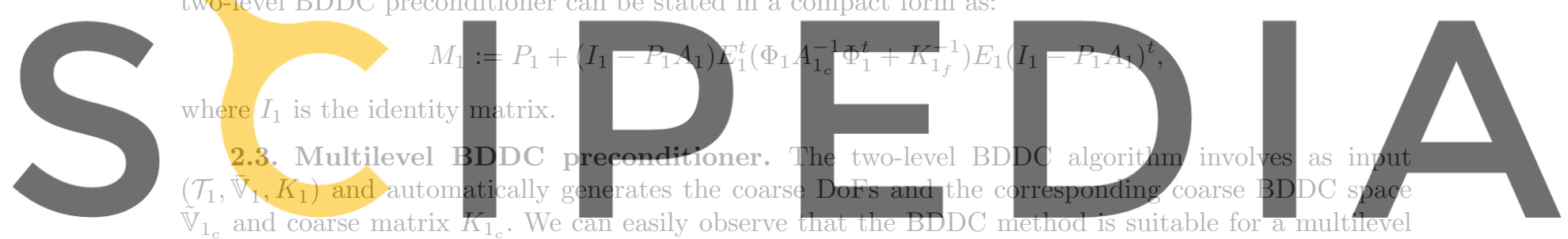

generalization (see [32] for three-levels and [25] for the general case). We can consider a coarse partition

portion of the coarse system matrix is at our disposal via the assembly of local sub-assembled matrices $K_{1}^{i}$ for subdomains $\Omega_{1}^{i} \in \mathcal{T}_{1}$ such that $\Omega_{1}^{i} \subseteq \Omega_{2}^{j}$, in the same fashion as above with FE matrices. Further, the coarse space $\tilde{\mathbb{V}}_{1_{c}}$ is a FE-like space associated to $\mathcal{T}_{1}$, and we can also generate its corresponding space of discontinuous functions with respect to $\mathcal{T}_{2}$. Therefore, we can readily define the BDDC preconditioner associated to the coarse system matrix $K_{1_{c}}$, the coarse space $\tilde{\mathbb{V}}_{1_{c}}$, and the partition $\mathcal{T}_{2}$. In the sequel, we express the multilevel method in a formal way.

We create a hierarchy of quasi-uniform partitions $\left(\mathcal{T}_{1}, \mathcal{T}_{2}, \ldots, \mathcal{T}_{n_{\ell}-1}\right)$ by aggregation, where $n_{\ell}$ is the number of levels in the BDDC method. $\mathcal{T}_{\ell+1}$ is a partition of $\mathcal{T}_{\ell}$. For every subdomain $\Omega_{\ell}^{i} \in \mathcal{T}_{\ell}$ there is only one $\Omega_{\ell+1}^{j} \in \mathcal{T}_{\ell+1}$ such that $\Omega_{\ell}^{i} \subset \Omega_{\ell+1}^{j}$, and we will denote $j$ as fat $(\ell, i)$. We can readily use all the notation in Sect. 2.2 just replacing 1 by $\ell$.

As input of the MLBDDC method, we require the sub-assembled subdomain matrix $K_{1}$ related to $\mathcal{T}_{1}$, the global FE space $\overline{\mathbb{V}}_{1}$, and the hierarchical partitions $\left(\mathcal{T}_{1}, \mathcal{T}_{2}, \ldots, \mathcal{T}_{n_{\ell}-1}\right)$. The set-up of the MLBDDC preconditioner is as follows:

For $\ell=1, \ldots, n_{\ell}-1$,

- Set-up $\left(P_{\ell}, K_{\ell_{f}}^{-1}, K_{\ell_{c}}, \Phi_{\ell}, E_{\ell}\right)$ using the procedure described in Sect. 2.2 replacing $\left(\mathcal{T}_{1}, \overline{\mathbb{V}}_{1}, K_{1}\right)$ by $\left(\mathcal{T}_{\ell}, \overline{\mathbb{V}}_{\ell}, K_{\ell}\right)$

- If $\ell==n_{\ell}-1$

- Else

$$
\text { * Set-up } A_{n_{\ell}-1}^{-1}
$$

* Initialize the next level with $\overline{\mathbb{V}}_{\ell+1} \equiv \tilde{\mathbb{V}}_{\ell_{c}}, K_{\ell+1} \equiv K_{\ell_{c}}$

After all these operators are set-up, we are in position to define the MLBDDC preconditioner $M$ in a recursive way as follows: $M \equiv M_{1}$ where

$M_{\ell}=P_{\ell}+\left(I_{\ell}-P_{\ell} A_{\ell}\right) E_{\ell}^{t}\left(\Phi_{\ell} M_{\ell+1} \Phi_{\ell}^{t}+K_{\ell_{f}}^{-1}\right) E_{\ell}\left(I_{\ell}-P_{\ell} A_{\ell}\right)^{t}$, for $\ell=1, \ldots, n_{\ell}-1$, and $M_{n_{\ell}}=A_{n_{\ell}-1_{c}}^{-1}$. 
We refer to [25] for a proof of the following theorem, about the condition number of the BDDCpreconditioned system matrix. We note that the results in [25] have been originally proved for the Laplacian problem. It can be extended to the linear elasticity case by combining the two-level bound for the condition number of BDDC and FETI-DP methods for 3D linear elasticity (see, e.g., [21]) with the multilevel analysis in [25].

THEOREM 2.1. The condition number of the BDDC preconditioned system matrix for the Laplacian and linear elasticity problem is bounded as

$$
\kappa(M A) \leq C \prod_{\ell=1}^{n_{\ell}-1}\left(1+\log \left(\frac{h_{\ell}}{h_{\ell-1}}\right)\right)^{2},
$$

for $B D D C(c)$ or $B D D C(c e)$ in $2 D$, and $B D D C(c e)$ and $B D D C(c e f)$ in $3 D$, where $C>0$ is a constant that does not depend on the hierarchical partition, i.e., number of levels $n_{\ell}$ and characteristic sizes.

3. An extreme scale parallel distributed-memory implementation. In this section we cover in detail a novel approach for the parallel distributed-memory implementation of the MLBDDCPreconditioned Conjugate Gradient (PCG) algorithm. In Sect. 3.1, we present the rationale underlying the novel approach that we pursue for the extreme scale implementation of this algorithm. In Sect. 3.2 we cover the building blocks of the parallel algorithm subject of study. In Sect. 3.3 we discuss a use case that illustrates how the techniques proposed are applied to a three-level BDDC-PGC solver in order to reach maximum performance benefit. Finally, in Sect. 3.4 we analyze how to choose values for the coarsening ratios governing subdomain aggregation in order to let the techniques proposed to be fully
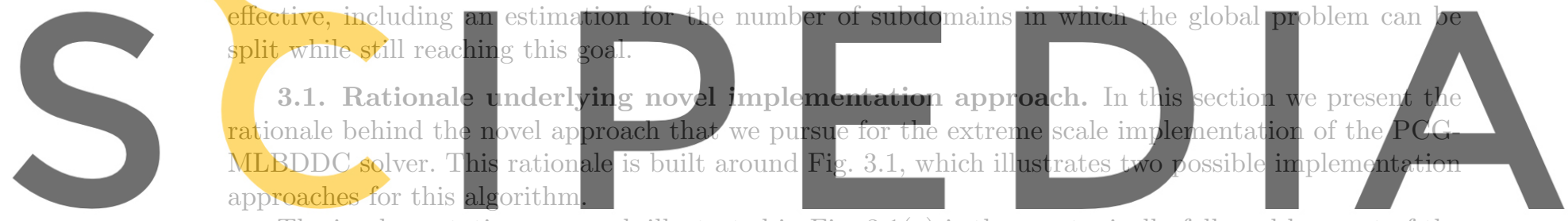

The implementation approach illustrated in Fig. 3.1(a) is the one typically followed by most of the Register foxisting code implementations of (ML)BDDC and related DD algorithms [8, 26, 29]. It comes naturally

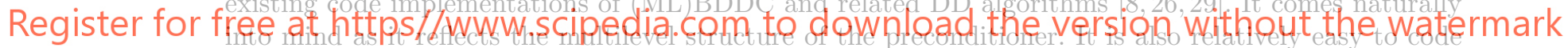

due to its bulk-synchronous structure. However, it does not exploit all the parallelism which is readily available in the algorithm as it serializes the computation and application of the MLBDDC hierarchy. For example, as discussed in Sect. 2, the fine and coarse-grid correction can be computed in parallel due to the $K$-orthogonality constraint underlying the BDDC space. Many other opportunities for parallelism are not exploited by this implementation approach. (See Sect. 3.3 for a full coverage of such opportunities in the case of a three-level MLBDDC-PCG solver.) As a first side effect, there is a significant loss of parallel efficiency due to idle MPI tasks. Due to the aggressive coarsening of these methods, the number of tasks at level 1 is much larger than those at higher levels. Thus, in such implementations, there is a notable aggregated idling time roughly equal to the number of cores being used times the time spent at levels higher than 1. It also has a negative impact in the energy consumption of such implementations. As a second side effect, the memory available on the core responsible for the coarsest-grid problem has to be shared among data structures corresponding to all levels. Provided the already very limited memory per core on current (and future) multicore-based massively parallel processors (e.g., it is only 1GB for the IBM BG/Q supercomputer), this limits even more the load per core that fits into memory (and thus that of the global problem size).*

Fig. 3.1(b) illustrates the novel implementation approach that we propose in this paper. It pursues full exploitation of the parallelism available in the algorithm. In order to reach this goal, the global MPI communicator is split into as many disjoint subsets of MPI tasks, i.e., subcommunicators, as levels in the MLBDDC preconditioning hierarchy. All MPI tasks are only in charge of a single subdomain at a particular level. Besides, the steps of the algorithm are re-organized (i.e., re-scheduled) in such a way that computation and communication in the global critical path is performed/issued as soon

${ }^{*}$ We note that a distributed-memory solver for the coarsest-grid problem $[16,26,29]$ can mitigate the impact of these side effects, but the parallel overhead due to idle MPI tasks still remains. 


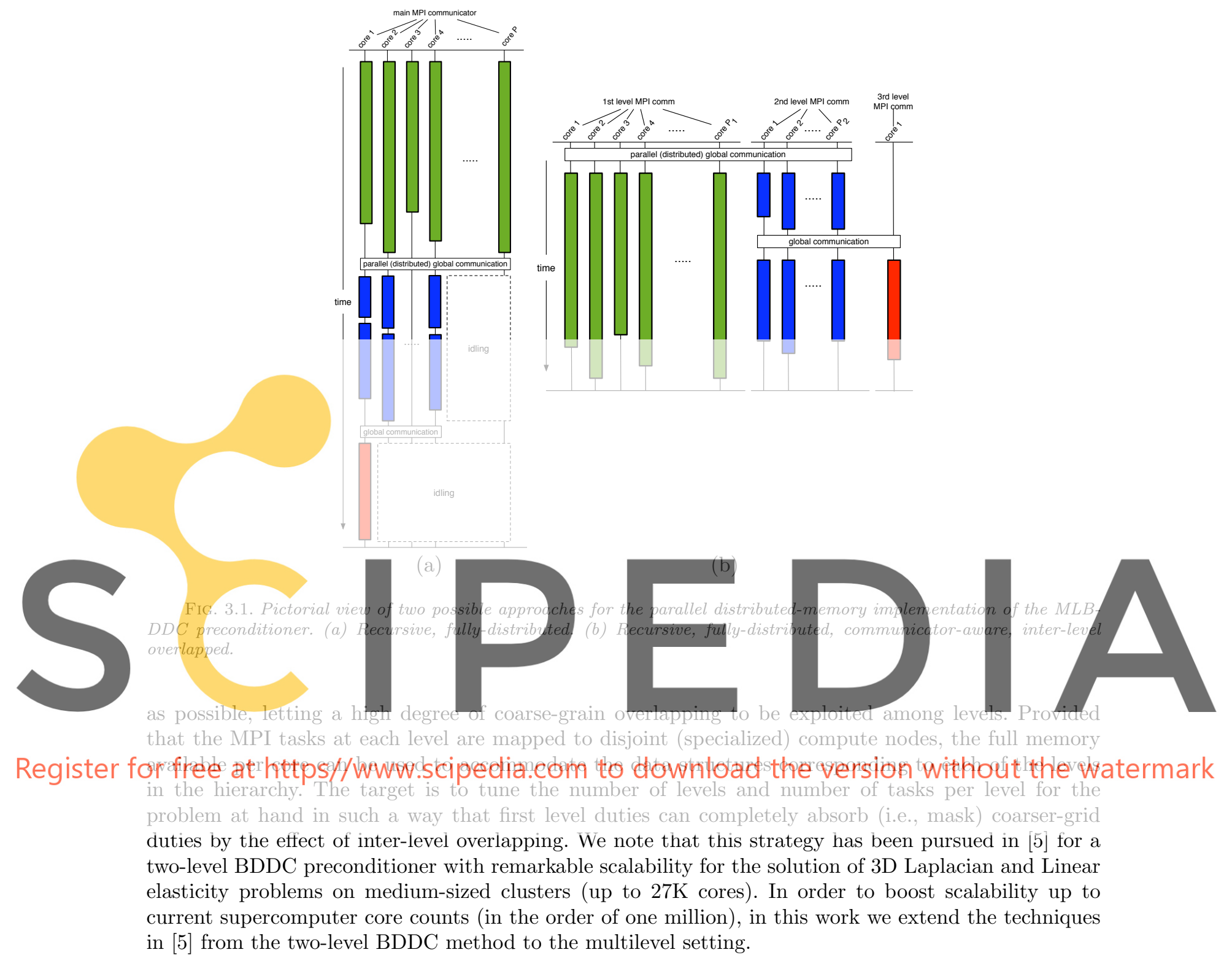

3.2. Building blocks. In Alg. 1 we present the main phases of the parallel distributed-memory solution of the linear system (2.1) via the MLBDDC-PCG solver. We can distinguish an initial phase encompassing lines 1-2 of Alg. 1, where the MLBDDC preconditioner is set-up, and an iterative phase in line 6, where the PCG solver is accelerated by means of the MLBDDC preconditioner. The PCG consists of a repeated sequence of the following four basic operations: application of the preconditioner, sparse matrix-vector products, inner products and vector updates [27].

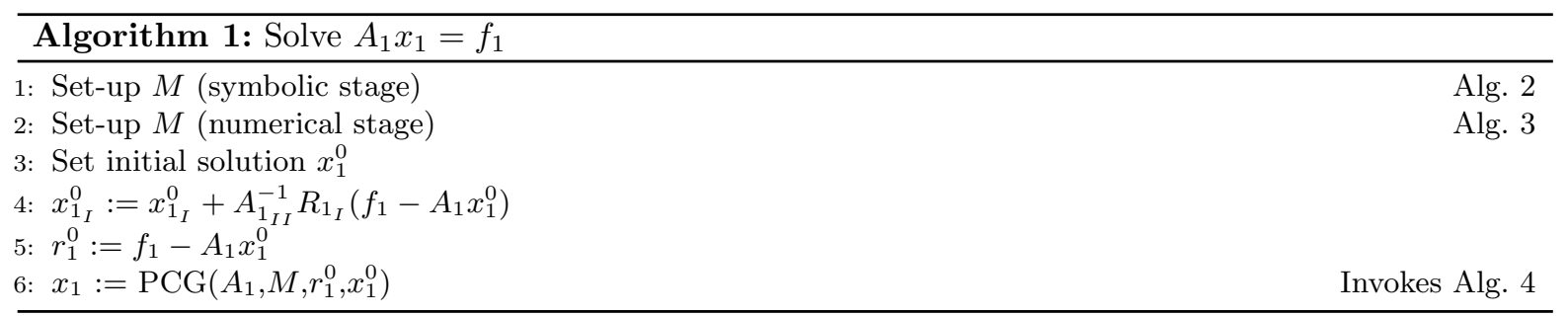

In the message-passing implementation of Alg. 1, all matrices, vectors, and associated computations 
are distributed among MPI tasks conformally with the hierarchy of non-overlapping partitions underlying the MLBDDC preconditioner. Let us denote by $\operatorname{task}(\ell, i)$ the one-to-one mapping that assigns a MPI task to every subdomain $\Omega_{\ell}^{i} \in \mathcal{T}_{\ell}$, for every level $\ell$. Any vector $x_{\ell} \in \mathbb{V}_{\ell}$ is distributed conformally with $\mathcal{T}_{\ell}$, i.e., the portion $x_{\ell}^{i}$ of $x_{\ell}$ is stored in MPI task task $(\ell, i)$. Analogously, matrix $K_{\ell}$ is distributed such that every diagonal block $K_{\ell}^{i}$ is stored in MPI task $\operatorname{task}(\ell, i)$. Vectors in $\overline{\mathbb{V}}_{\ell} \subset \mathbb{V}_{\ell}$ are distributed as those in $\mathbb{V}_{\ell}$, with the particularity that they must be continuous on the interface, i.e., values on interface DoFs must match among MPI tasks sharing them. As a result, data structures describing the distributed-memory layout of such vectors must provide additional gluing information. We refer the reader to [4] for a comprehensive coverage of such data structures in a two-level BDDC preconditioner context. The discussion there straightforwardly applies to the MLBDDC preconditioner.

In Algs. 2-3 we collect all the steps required to set up the MLBDDC preconditioner, while those required for its application to a residual vector at each PCG iteration are shown in Alg. 4. In this work we consider sparse direct methods [12] for the exact (up to machine precision) solution of the subproblems within the MLBDDC preconditioning hierarchy. Indeed, the reader might have already observed that Algs. 2, 3 and 4 match the stages involved in the direct solution of sparse linear systems. In particular, preconditioner set-up is split into a symbolic stage in Alg. 2 (with $G_{C}$ denoting the graph which describes the sparsity pattern of matrix $C$ ), followed by a numerical stage in Alg. 3 . Alg. 2 essentially builds and symbolically factorizes the graph associated to local matrices $A_{\ell_{I}}^{i_{\ell}}, K_{\ell_{f}}^{i_{\ell}}$, for $\ell=1, \ldots, n_{\ell}-1, i_{\ell}=1, \ldots, n_{\ell}^{\text {sbd }}$, and that of the global coarsest-grid matrix $A_{n_{\ell}-1_{c}}$. As part of the symbolic analysis, a fill-in reordering is applied to the graph. On the other hand, Alg. 3 is in charge of the numerical factorization of these matrices. Once the sparse Cholesky factor of (each of) these matrices is set-up, Alg. 4 solves the corresponding linear systems required for the application of
MLBDDC preconditioner hierarchy by means of sparse forward/backward substitution. We note that
the Dirichlet pre-correction in Alg. 4 sed lines 2 and 3) can be omitted at the first level, since the
interior residual is already ze-o due to the initial interior pre-correction (see line 4 of Alg. 1).
Algorithm 2: MMLBDDC set-up (symbolic stage)

Reord + Symb fact $\left(G_{A_{\ell_{I}}^{i}}\right)$

Identify local coarse DoFs ipedia.com to download the version without the watermark

4: Gather coarse-grid DoFs

: if $\ell==n_{\ell}-1$ then

6: $\quad$ Build $G_{A_{n_{\ell}-1}}$

7: $\quad$ Reord+Symb fact $\left(G_{A_{n_{\ell}-1_{c}}}\right)$

$\ell \rightarrow \ell+1$

: else

9: $\quad$ Build $G_{K_{\ell_{c}}^{j}}$

10: $\quad$ Define $G_{K_{\ell+1}} \leftarrow G_{K_{\ell_{c}}}$ and invoke Alg. 2 with $\ell \leftarrow \ell+1$ end

Let us finally describe the meaning of labels at the end of the steps in Algs. 2, 3 and 4. On the one hand, MPI tasks $\operatorname{task}(\ell, \cdot)$ are in charge of the steps labeled as " $\ell$ ", while MPI tasks $\cup_{k=\ell+1}^{n_{\ell}} \operatorname{task}(k, \cdot)$ perform those labeled as " $\ell+1, \ldots, n_{\ell}$ ". Note that in Algs. 2,3 , and 4 we are assuming, without loss of generality, that $A_{n_{\ell}-1_{c}}$ is centralized on a single MPI task at the last level (so that a serial sparse direct solver can be used for the solution of the coarsest-grid problem). However, this problem can be also distributed among several MPI tasks (and solved by means of a message-passing sparse direct solver as the one in MUMPS [2]). On the other hand, labels " $\ell \rightarrow \ell+1$ " refer to data transfers among MPI tasks in two consecutive levels. More precisely, among level $\ell$ MPI tasks, task $(\ell, i)$, and their parents at level $\ell+1$, i.e., task $\operatorname{task}(\ell+1, j)$ such that $j=\operatorname{fat}(\ell, i)$.

3.3. Use case for a three-level BDDC-PCG solver. According to Sect. 3.1, the steps in Algs. 2-4 have to be judiciously re-organized in order to fully exploit all parallelism which is readily available in these algorithms. Table 3.1 depicts the result of this exercise for a three-level BDDCPCG solver. (We have considered a three-level algorithm for the sake of simplicity when discussing the 

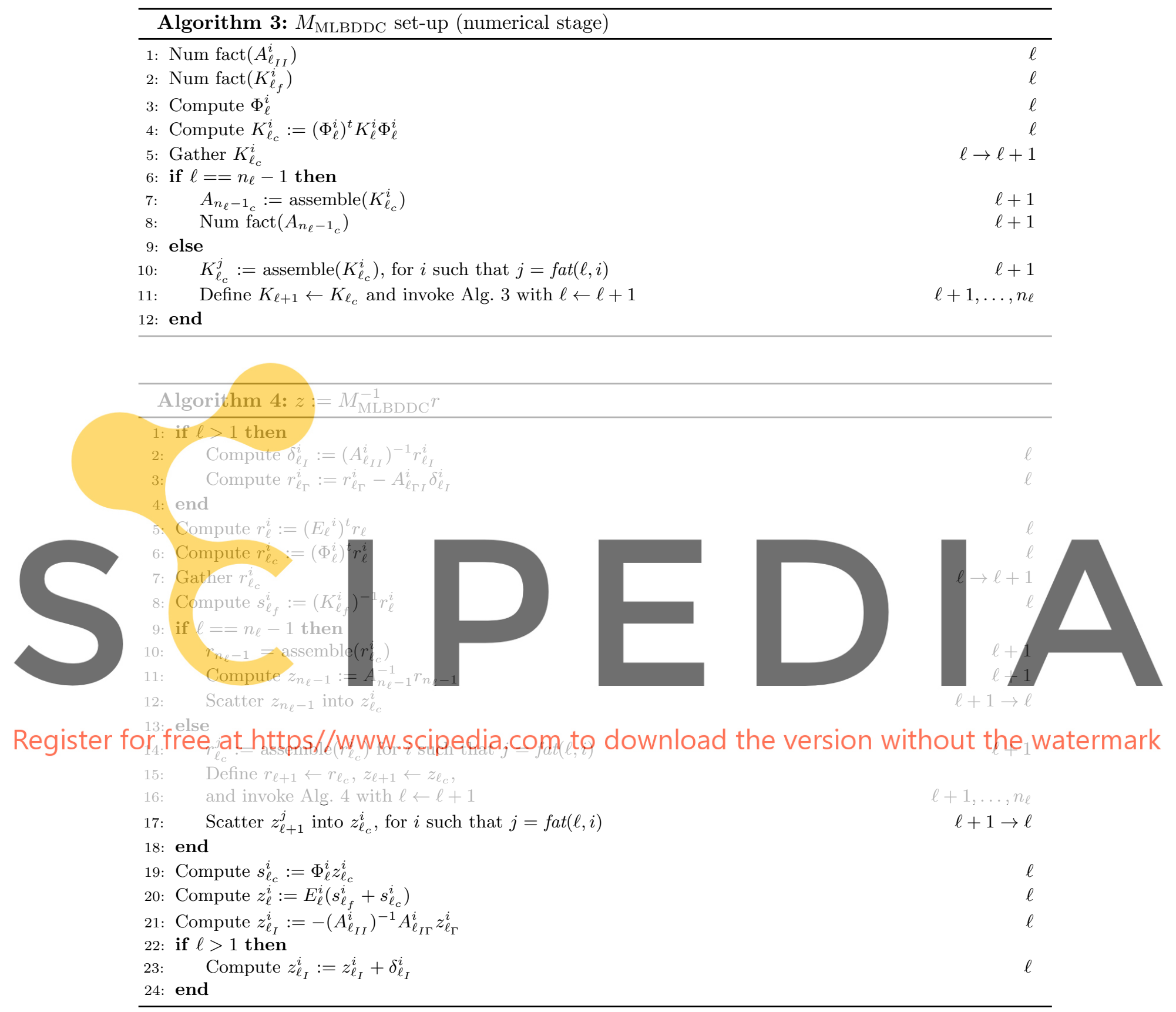

overlapping potential of our approach, even though the implementation of the algorithm is recursive and can be applied to an arbitrary number of levels; see Sect. 3.4 and 4.) In this table, the steps to be performed have been grouped into coloured regions in order to clarify the exposition. In particular, green regions encompass local computations and nearest neighbor communications residing at the first level, while blue regions those at the second level. There are three of such green and blue regions separated by gather and scatter communication stages among first and second level MPI tasks (uncolored at the table). Blue regions are in turn split by communication stages among second and third level MPI tasks (colored in gray). Finally, red regions include computations at the third level separated by the latter communication stages.

Let us now characterize the balance that has to be struck among the time spent in the colored regions of Table 3.1 in order to let the techniques proposed to be fully effective. To do such characterization, 


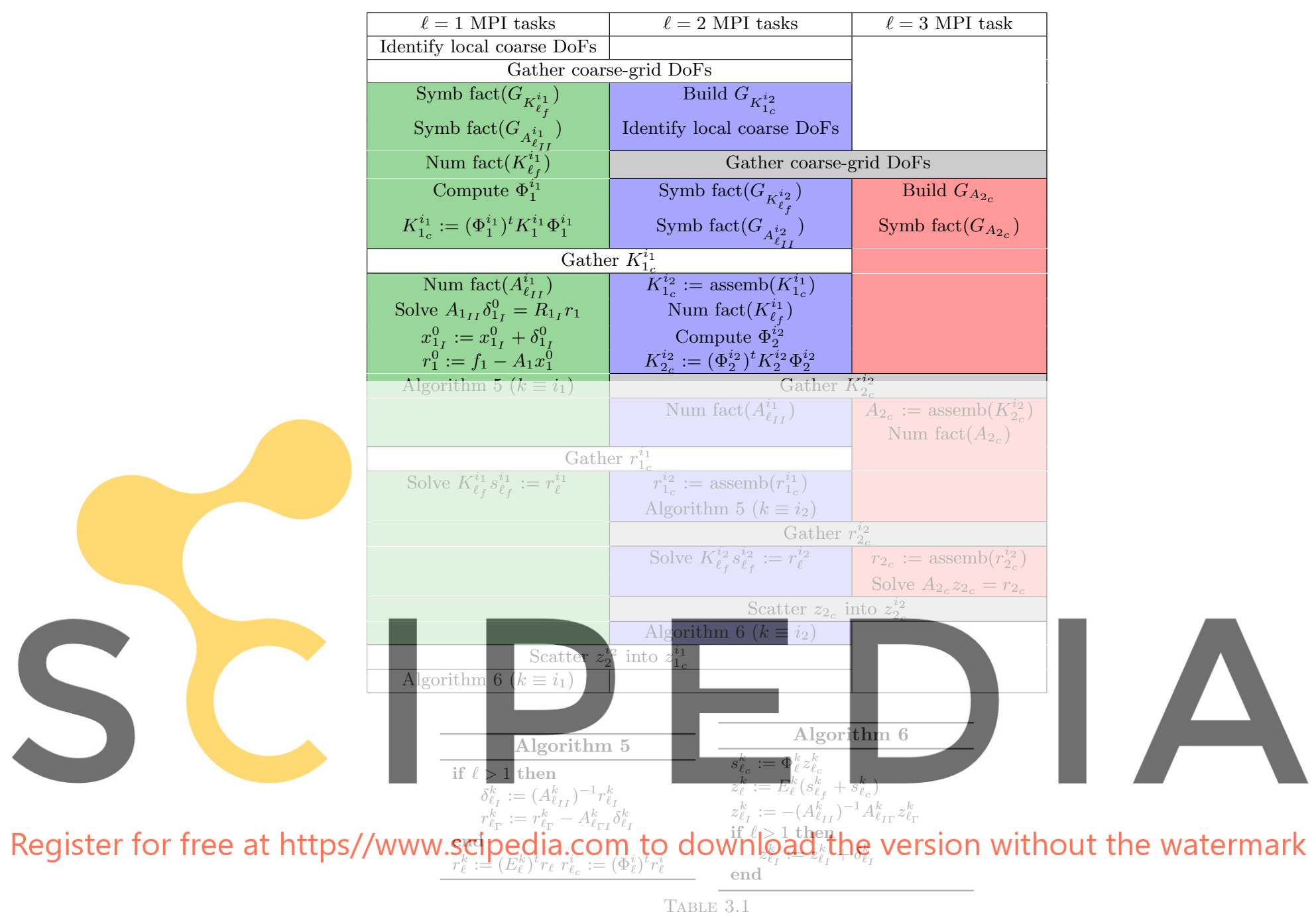

Mapping of Algs. 2-4 to MPI tasks that maximizes inter-level overlapping for a three-level BDDC-PCG solver.

we take as basis a key observation derived from the table: full effectiveness is fulfilled if and only if the MPI tasks at the second level issue the gather and scatter communication operations among first and second level MPI tasks at most at the same time as those in the first level. Any delay of second level MPI tasks in reaching these communication stages (with respect to first level MPI tasks) immediately implies parallel efficiency loss. In such situation (we will later analyze on which factors does it depend to get it), the steps encompassed in green areas will fully absorb (i.e., mask) the latency associated to coarser-grid level duties (i.e., blue and red regions in Table 3.1). The reader should note at this point that this scenario leads to remarkable performance and scalability, provided that steps encompassed in green regions are of local nature, therefore fully parallel.

An obvious necessary but not sufficient condition to achieve full effectiveness is that the steps encompassed by each of the three blue regions at the second level take less time than those at the corresponding green regions. Let us discuss on which factors does this depend for each of the three green/blue region pairs separately, restricting ourselves to the most computationally-dominant steps:

- In the first green/blue region pair, the symbolic factorization of the graph associated to Dirichlet and constrained Neumann problems, the numerical factorization of a constrained Neumann problem, and the computation of $\Phi_{1}^{i_{1}}$ at the first level, should take at least the same time as the former two symbolic factorizations at the second level. Provided that numerical factorization has higher order of complexity than symbolic factorization (see Sect. 3.4), this goal is relatively 
easy to achieve.

- In the second green/blue region pair, the numerical factorization of the Dirichlet problem matrix, and the solution of a linear system with this matrix, should take at least the same time as the numerical factorization of the constrained Neumann and Dirichlet problems matrices and the computation of $\Phi_{2}^{i_{2}}$ at the second level. Provided that there are two numerical factorizations at the blue region per one at the green one, it becomes harder to achieve our goal in this case. This is is still feasible though, e.g., by enforcing sufficiently smaller subdomain sizes at the second level compared to the sizes of the ones at the first level.

- Analogously, in the last green/blue region pair, the solution of a constrained Neumann problem at the first level should at least take the same time as the solution of two Dirichlet problems (see Alg. 5 and 6), and a Neumann constrained problem at the second level.

At this point, we note that blue regions are in turn split by communication stages among second and third level MPI tasks (colored in gray in Table 3.1). Therefore, if the time spent in red regions becomes "large enough", then communication stages below these red regions can in turn delay second level MPI tasks with respect to first level ones up to an extent that starts threatening the full effectiveness of this approach. In fact, it irremediably happens at some point when the number of MPI tasks at the coarsest level is kept fixed in a weak scaling analysis. In order to analyze this effect, we split again the discussion among overlapping regions, in order to infer how the coarsest level duties can harm the effectiveness of the overlapping between first and second level MPI tasks. We focus on the second and third region since there is no potential level-2 idling in the first overlapping green/blue area:

- When the symbolic factorization of the level-3 coarse matrix in the first red region becomes dominant, i.e., it cannot be masked by the symbolic factorization of the graph associated to Dirichlet and constrained Neumann problems and the numerical factorization of a constrained Neumann problem in the second blue region, level-2 MPI tasks have to wait at the corresponding gather communication. As a result, the first red region (level-3) can potentially degrade the effectiveness of the second green/blue overlapping area.

- When the numerical factorization of the coarsest matrix (in charge of level-3 MPI tasks) cannot be masked by the numerical factorization and triangular solve of the second and third blue regions, level-2 tasks have to wait in the corresponding gather communication. Idling of level-2 MPI tasks also occurs when the third red region (one sparse backward/forward solve) cannot be masked by the three sparse backward/forward solves in the third blue region. As a result, the second and third red regions (level-3) can potentially degrade the effectiveness of the third green/blue overlapping area.

In a weak scaling scenario, the subdomain problem size at the first and second levels is fixed. On the other hand, for a fixed number of MPI tasks at the third level, the third level local problem size increases with the number of subdomains at the second level. As a result, to maximize the scalability of the method, it is interesting to make subdomain coarsening from the first to the second level as aggressive as possible, but always keeping second level (blue) regions masked by first level (green) ones. If this strategy does not allow to achieve full effectiveness within the whole range of core counts of interest, a possible solution is to introduce an additional level in the hierarchy to ameliorate the growth of the time spent in red regions.

We stress that the discussion in this section applies up to minor details to MLBDDC preconditioning hierarchies with an arbitrary number of levels. At any pair of intermediate levels (1-2, 2-3, 3-4, etc.), one has to deal with pairs of regions very close to those green/blue region pairs in Table 3.1 (up to the fact that the input data structures of first level MPI tasks are provided by a FE integration module, instead of being gathered from the previous level). Any of the regions at the intermediate levels (included the ones at the last level) have the potential of threatening full effectiveness if the time spent on any of them becomes "sufficiently large", in a some sort of delay-ripple effect that finally causes first level MPI tasks having to waste time on the communication stages among first and second level MPI tasks. In any case, the effect coarsest-level duties have in green/blue region pairs is the same regardless of the number of levels.

3.4. Estimates for effective coarsening ratios. In this section we provide a comprehensive discussion about an effective choice of the coarsening ratios governing subdomain aggregation among partitions at different levels, in order for the overlapped multilevel implementation to be fully effective. 
It depends on the number of levels being used and the subdomain size at every level, which in turn depends on the coarsening ratios being used in the definition of the hierarchical partitions.

Before entering upon the subject, let us recall the complexity of the main stages of the direct solution of sparse linear systems. In sparse direct methods, provided that a 3D problem is being solved, the symbolic factorization has a cost $c_{\mathrm{sf}} n^{\frac{4}{3}}$, the numerical factorization a cost $c_{\mathrm{nf}} n^{2}$, and the triangular system solve a cost $c_{\mathrm{ts}} n^{\frac{4}{3}}$, where $n$ is the size of the coefficient matrix, and the constants $c_{\mathrm{sf}}$, $c_{\mathrm{nf}}$, and $c_{\mathrm{ts}}$ do depend on the particular algorithms, their implementation, and the underlying hardware.

Let us now enter upon the subject, and consider partitions that are well-balanced at all levels, i.e., all subdomains at the same level have (roughly) the same number of elements. We denote by $m_{\ell}$ (resp. $N_{\ell}$ ) the local (resp. global) number of elements at level $\ell$, and by $p_{\ell}$ the number of subdomains at level $\ell$. We note that $N_{\ell+1}=p_{\ell}$, and that $m_{\ell}=\frac{N_{\ell}}{p_{\ell}}=\frac{p_{\ell-1}}{p_{\ell}}=\frac{N_{\ell}}{N_{\ell+1}}=\left(\frac{h_{\ell+1}}{h_{\ell}}\right)^{d}$.

In a weak scaling scenario of one-level algorithms, the global problem size $N_{1}$ and the number of subdomains $p_{1}$ with level- 1 duties increase in such a way that $m_{1}=\frac{N_{1}}{p_{1}}$ is kept fixed. For a multilevel algorithm, we aim to keep the effectiveness of the preconditioner in terms of iteration counts, which requires to keep the bound of the condition number given by Theorem 2.1 constant. Thus, we enforce that the local number of elements $m_{\ell}=\frac{p_{\ell-1}}{p_{\ell}}$ is fixed at levels $\ell=1, \ldots, n_{\ell}-2$, which involves to increase $p_{\ell}$ accordingly. Level $n_{\ell}$ is assumed to be mapped to one (or at least to a fixed) number of MPI tasks.

Given $m_{\ell}$ (number of local mesh elements, where we denote by elements the subdomains of the previous level), the actual size of the local system is denoted by $\beta_{\ell} m_{\ell}$ (number of local DoFs), where $\beta_{\ell}$ is affected by the type of DoFs in the BDDC method for $\ell>1$ or by the order of the FE space for $\ell=1$.

Let us assume that we keep fixed the number of levels $n_{\ell}$ in a weak scalability analysis. The questions that arise are: How do we choose the coarsening ratios between levels to attain optimal scalability? Fixing a hierarchical partition, at which number of MPI tasks the overlapping strategy will stop being effective?

The definition of the hierarchical partition can be parametrized by the desired number of elements per level, namely $m_{1}, \ldots, m_{n_{\ell}-1}$. In order to answer (qualitatively) the former questions, let us make two assumptions:

1. The bulk of the CPU time in the overlapped regions is spent at the different phases of the sparse direct solvers (symbolic factorization, numerical factorization, triangular system solves). Nearest-neighbor communications at level 1 , all communications among levels $\ell=2, \ldots, n_{\ell}$, and other steps are assumed to be negligible.

2. The cost of the phases of a sparse direct method when applied to the constrained Neumann problem is assumed to be identical to that of the corresponding ones involved in the solution of the Dirichlet problem.

The validity of the first assumption increases with the local problem size, but it is mild for reasonably loaded cores (about $256 \mathrm{~KB}$ of memory per core), due to the low CPU cost (and excellent scalability) of nearest-neighbor communications and the reduced number of cores at levels greater than one. The second assumption is also reasonable, since both problems have almost the same size.

Taking into account the steps in each of the three green/blue region pairs in Table 3.1, their complexities (see above), and the previous assumptions, we easily infer that the steps at level $\ell+1$ are fully masked by level- $\ell$ steps if the following over-pessimistic condition holds:

$$
c_{\mathrm{nf}}\left(\beta_{\ell} m_{\ell}\right)^{2}+2 c_{\mathrm{ts}}\left(\beta_{\ell} m_{\ell}\right)^{\frac{4}{3}} \geq 2 c_{\mathrm{nf}}\left(\beta_{\ell+1} m_{\ell+1}\right)^{2}+n_{\Phi} c_{\mathrm{ts}}\left(\beta_{\ell+1} m_{\ell+1}\right)^{\frac{4}{3}},
$$

which corresponds to the second and third green/blue regions in Table 3.1, where $n_{\Phi}$ denotes the maximum number of coarse DoFs per subdomain. This is a sufficient condition to fully mask coarse duties by level-1 (fine) duties, but by any means necessary.

As commented above, in a weak scaling scenario and a fixed number of levels, the coarsest-grid related steps CPU time will increase. Scalability loss will only affect the overall execution time when level-1 regions cannot mask the ones at level 2. Considering the effect that the coarsest level has in level-1 regions (see above), we get the following additional sufficient (over-pessimistic) conditions to fully mask coarse duties by level-1 (fine) duties:

$$
\beta_{1} m_{1} \geq\left(\frac{c_{\mathrm{sf}}}{c_{\mathrm{nf}}}\right)^{\frac{1}{2}}\left(\beta_{n_{\ell}} m_{n_{\ell}}\right)^{\frac{2}{3}}, \quad\left(\beta_{1} m_{1}\right)^{\frac{4}{3}} \geq\left(\beta_{2} m_{2}\right)^{\frac{4}{3}}+\left(\beta_{n_{\ell}} m_{n_{\ell}}\right)^{\frac{4}{3}}+\left(\frac{c_{\mathrm{nf}}}{c_{\mathrm{ts}}}\right)\left(\beta_{n_{\ell}} m_{n_{\ell}}\right)^{2},
$$


which correspond to the second and third region, respectively. We can easily observe out of these constraints that the most challenging one is the latter, because we have to mask steps with higher complexity. Let us consider the worst-case scenario in which the last level is centralized on a single MPI task. The number of elements at the last level will increase in a weak scaling scenario as follows:

$$
m_{n_{\ell}}=p_{n_{\ell}-1}=\frac{p_{n_{\ell}-2}}{m_{n_{\ell}-1}}=\ldots=\frac{p_{1}}{m_{n_{\ell}-1} \cdots m_{2}} .
$$

Replacing (3.3) in (3.1) and (3.2), we get an indication about the maximum number of cores that can be used before loosing parallel efficiency. However, in order to use this formula, we need to provide a value for the ratios $\frac{c_{\mathrm{ts}}}{c_{\mathrm{nf}}}$ and $\frac{c_{\mathrm{sf}}}{c_{\mathrm{nf}}}$. We estimated this constant by regression for the sparse direct solver codes and computer architecture used in Sect. 5 for the 3D Laplacian problem for five structured cubic meshes (from 4, 096 to $512,000 \mathrm{FEs}$ ), getting $\frac{c_{\mathrm{ts}}}{c_{\mathrm{nf}}} \approx 400$ and $\frac{c_{\mathrm{sf}}}{c_{\mathrm{nf}}} \approx 500$. These large values are expected, since the cost per flop for numerical factorization is low, due to the intensive use of the cache hierarchy by means of the Level 3 BLAS, whereas the triangular solution and symbolic factorization are memory-bounded.

Let us consider two practical examples from Sect. 5. In 3D, and linear FEs, a local problem size per subdomain of $m_{1}=20^{3}$ fits into the $1 \mathrm{~GB}$ of memory available per core. $\beta \approx 1$ for linear FEs, and $\beta \approx 4$ for BDDC(ce) when solving the Laplacian problem. In this case, taking as an example a three-level BDDC(ce) method with $m_{2}=7^{3}$, condition (3.1) easily holds whereas condition (3.2) combined with (3.3) leads to $p_{1} \lesssim 422,919$ subdomains. A four level algorithm with $m_{1}=20^{3}, m^{2}=4^{3}$, and $m^{3}=3^{3}$ leads to $p_{1} \lesssim 2,252,500$. For linear elasticity, where the values of $\beta$ have to be multiplied by a factor of three, the last case leads to $p_{1} \lesssim 1,714,300$. These bounds are in agreement with the numerical results obtained in Sect. 5 for these combinations, and are an indication of the potential of the strategy presented so far.

4. Code implementation details. In this section, we sketch, thorough (simplified) Fortran95 code snippets, some key hints for the MPI-parallel implementation of the approach presented in Sect. 3. In particular, Listing 1 sketches the initialization of the MLBDDC preconditioner data structure (i.e., type (mlbddc) derived data type), while Listing 2, its numerical set-up stage (see Alg. 2). We stress that, for simplicity and brevity, many details related to software engineering practices in our software package (see Sect. 5) have been omitted, as e.g., those related with algorithm and code parameters, error and memory handling, or data encapsulation. Still, we expect these hints to be useful for practitioners willing to implement in their software the novel techniques proposed herein.

Let us start with the subroutine in Listing 1. This subroutine takes as input arguments a set of four MPI communicator handlers. comm_world is a communicator handler that includes all MPI tasks that contribute to the computation. (In most cases it will be mpi_comm_world, or more conveniently, a duplicate of it.) comm_l1 and comm_lgt1 are two communicator handlers for (disjoint) subcommunicators of comm_world which include the MPI tasks at the first level in the hierarchy, and those at higher levels, respectively. Finally, intcomm_l1_lgt1 is a handler for a MPI intercommunicator among the former and latter subcommunicators. This intercommunicator is not used for preconditioner set-up, but actually during the iterative solution phase. It allows (one of the) first level MPI tasks to signal (broadcast) higher levels tasks whether the iterative process converged (or not). We note that these four communicators are created outside the subroutine, in an initialization stage of our simulation software, and encapsulated in a derived data type which controls the MPI parallel environment. Any subroutine in the code needs access to this object to properly dispatch the path to be followed by each of the MPI tasks in comm_world. Finally, nlev, and tsks_per_lev dummy arguments refer to $n_{\ell}$, and $p_{\ell}$, for $\ell=1, \ldots, n_{\ell}-1$, respectively, while fat_map is an array that drives subdomain aggregation among levels. In particular, on input to the root call of this subroutine, fat map $(\ell)=\operatorname{task}(\ell+1, f a t(\ell, i))$ on MPI task task $(\ell, i)$. fat map is generated in preprocessing phase, when the partition of the FE mesh is performed, by (recursively) partitioning the graph of subdomains.

In lines 24-34 of Listing 1, comm_world is split into subcommunicators such that first level MPI tasks provide fat_map(1) as the color, while second level ones, their rank identifier in comm_12. The rest of tasks do not contribute to data transfers among the first and second level, and therefore provide color=mpi_undefined. Therefore, a subcommunicator is created for each subset of first and second level MPI tasks that have to exchange data. (Later on this section we will cover how to efficiently implement these data transfers.) In preparation to the recursive call, second level and higher level MPI tasks enter 


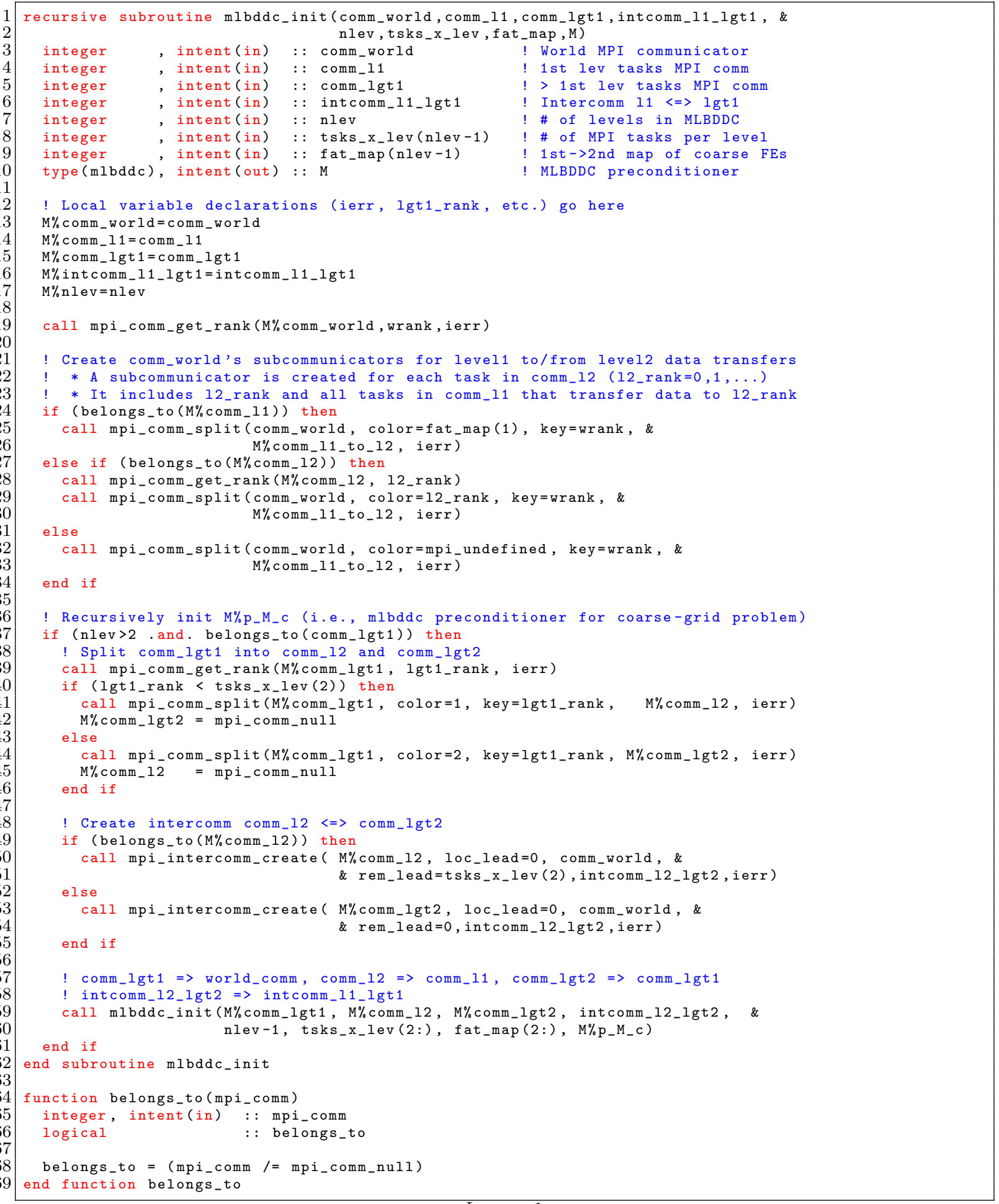

LISTING 1
Simplified Fortran95 MPI recursive subroutine in charge of preconditioner initialization.

lines 37-61, while those at the first level just exit the subroutine in the search of additional first level duties. The former set of tasks split comm_lgt1 into comm_12 and comm_lgt2 in lines 39-46, while an intercommunicator among comm_12 and comm_lgt2 is created in lines 49-55. This intercommunicator is required if the coarse-grid problem is to be solved, e.g., by a $\left(n_{\ell}-1\right)$-level BDDC-PCG or Richardson 


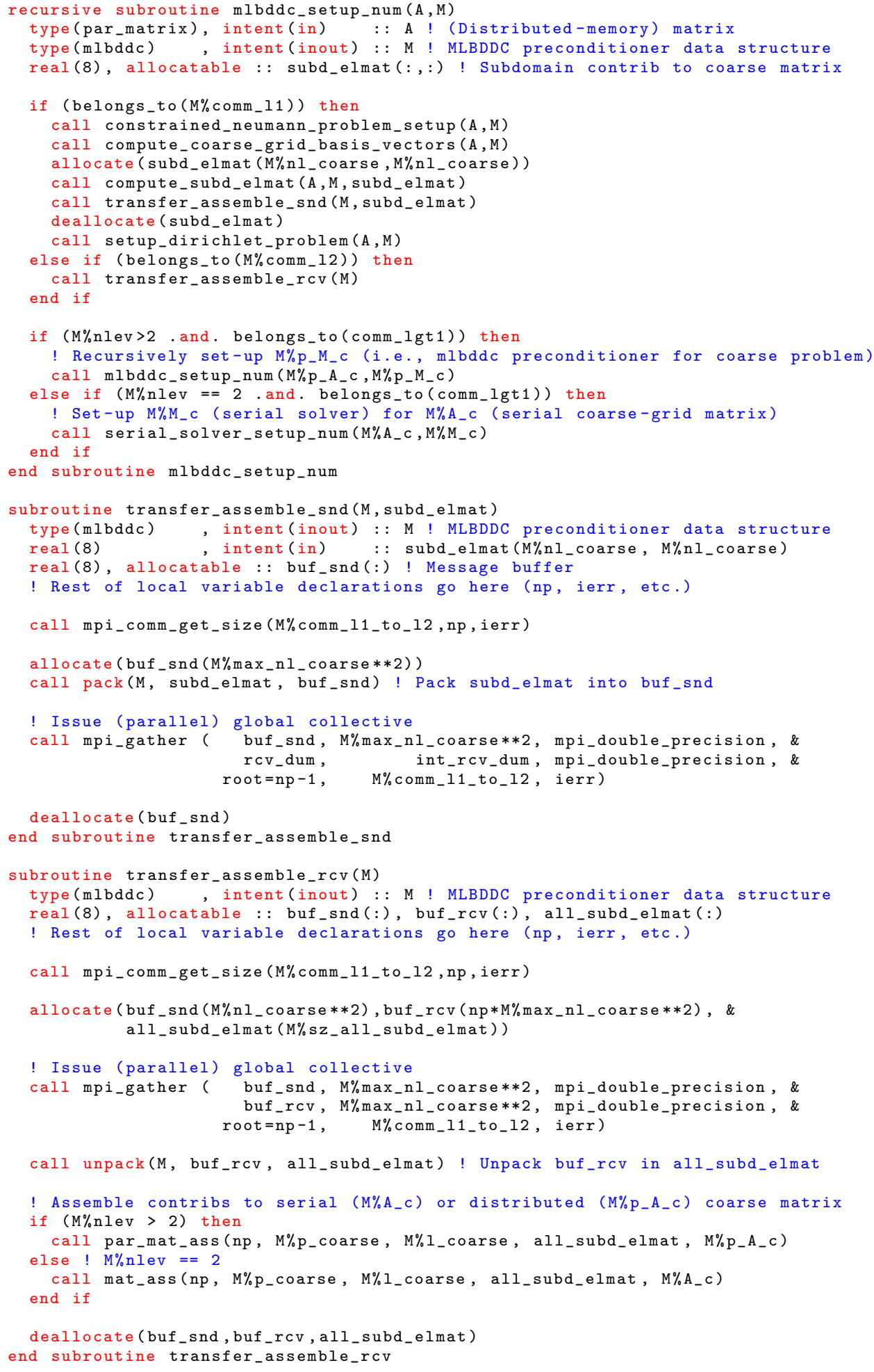

Simplified Fortran 95 MPI recursive subroutine in charge of preconditioner set-up (numeric).

iterative solver (instead of by a single application of this preconditioner). Finally, the data structure corresponding to the coarse-grid problem $n_{\ell}-1$-level BDDC preconditioner (i.e, M\%p_M_c) is initialized by 
a recursive call to $\mathrm{mlbddc}$ init in line 60 . Note that in the recursive call the set of four communicators (comm_lgt1, comm_12, comm_lgt2, intcomm_12_lgt2) play the role of (comm_world, comm_l1, comm_lgt1, intcomm_11_lgt1), respectively, on input to the recursive call.

The subroutine in charge of the numerical set-up stage of the MLBDDC preconditioner is shown in Listing 2. It takes as input an instance A of the type (par matrix) derived data type, and it sets up an instance $\mathrm{M}$ of the preconditioner data structure. The former derived data type internally accommodates a distributed sparse matrix, including the data describing its memory layout conformally with a nonoverlapping partition into subdomains. A first stage of preconditioner set-up encompasses lines 6- 16, and only involves first and second level MPI tasks. In particular, in lines 7 and 13 first level MPI tasks set up the local solver for the constrained Neumann and Dirichlet problems, respectively, and compute the coarse-grid basis vectors in line 8. In preparation for the coarse-grid problem matrix assembly, first level MPI tasks first compute subdomain contributions to the coarse-grid problem and store them in subd_elmat; see line 10. Then, first and second level MPI tasks enter transfer_assemble_snd and transfer_assemble_snd in lines 11 and 15, respectively. By means of these two subroutines, second level MPI tasks gather subdomain contributions from (their corresponding) first level MPI tasks. These contributions are then assembled in lines 64-68 of Listing 2. We note that these contributions are assembled in a type (par_matrix) instance M\%p_A_c, in case of any intermediate level of the hierarchy, or in a serial (centralized) sparse matrix instance $\mathrm{M} \% \mathrm{~A}_{-} \mathrm{c}$, at the end of the hierarchy (see lines 65 and 67 , respectively). A final stage of Listing 2 encompassing lines 18-24, recursively sets up, at any intermediate level, the $\left(n_{\ell}-1\right)$-BDDC preconditioner on second and higher level MPI tasks (see line 20), or sets up, at the end of the hierarchy, the serial solver instance $\mathrm{M}_{0} \mathrm{M} \_c$ for the coarse-grid problem matrix instance $\mathrm{M} \%$ A_c on the last level MPI task (see line 23).

The implementation of inter-level data transfers in Listing 2 deserves further attention. A subcommunicator for each subset of $\ell$ and $\ell+1$ level MPI tasks that have to exchange data was created during preconditioner initialization. Each set is defined as those $i$ having the same $f a t(\ell, i))$ together with $f a t(\ell, i)$ ) (their master) which permits the reuse of the code implementing a two-level BDDC in which the coarse solver is solved serially in a single separated MPI task [5]. These data transfers are implemented in lines 39 and 57 in such a way that multiple independent mpi_gather operations are issued simultaneously on each of these subcommunicators. By means of performance analysis tools, we could confirm that these implementation approach is able to efficiently exploit the underlying network hardware parallelism of the IBM BG/Q supercomputer. In particular, these communication stages take asymptotically constant time provided $\ell$ and $\ell+1$ level MPI tasks are scaled proportionally (i.e., weak scaling scenario). Another technical detail is that the code in Listing 2 exploits fixed message size collectives, instead of variable message sizes ones (mpi_gatherv), and therefore the actual (variable size) data to be sent, has to be packed to/unpacked from the (padded) message buffer on entry/exit to mpi_gather. Although our actual code provides both solutions, we observed in practice that fixed message-size collectives lead to much better performance/scalability (despite the overhead associated to padding).

Finally, we would like to stress that the mapping in Table 3.1 for a three-level BDDC-PCG solver (and the one corresponding to a MLBDDC preconditioner with an arbitrary number of levels) is not statically coded in the software, but instead results from the recurrent application of two techniques, namely recursion and communicator-awareness, in order to strategically deploy a different path for the MPI tasks residing at each level.

5. Numerical experiments. In this section, we study the weak scalability of the MLBDDC-PCG solver codes based on the implementation techniques presented in Sect. 3 and 4. As model problems, we consider the Laplacian (Sect. 5.2) and linear elasticity (Sect. 5.3) 3D PDEs on regular domains, discretized with structured (cartesian) FE meshes. We stress, however, that the algorithms and software are designed to handle arbitrary geometries and unstructured meshes as well. As performance metrics, we will focus in the number of PCG iterations required to converge, and the total computation time. In all the experiments reported in this section, this time will include both preconditioner set-up and the preconditioned iterative solution of the linear system (2.1).

5.1. Experimental framework. The novel techniques proposed in this paper for the MLBDDCPCG solver were implemented in FEMPAR. FEMPAR, developed by the members of the LSSC team at 
CIMNE, is a parallel hybrid OpenMP/MPI, object-oriented software package for the massively parallel Finite Element (FE) simulation of multiphysics problems governed by PDEs. Among other features, it provides the basic tools for the efficient parallel distributed-memory implementation of substructuring DD solvers [4]. The parallel codes in FEMPAR heavily use standard computational kernels provided by (highly-efficient vendor implementations of) the BLAS and LAPACK. Besides, through proper interfaces to several third party libraries, the local Dirichlet and constrained Neumann problems at each intermediate level of the hierarchy, and the global coarsest-grid problem at the last level, can be exactly solved via sparse direct solvers. In this work, we in particular explore HSL_MA87 [18], which provides a highly-efficient parallel multi-threaded DAG-based code implementation of the supernodal sparse direct Cholesky solver. The nested dissection algorithm available in METIS (v5.1.0) [19] was used as a fill-in reducing ordering for HSL_MA87. FEMPAR is released under the GNU GPL v3 license, and is more than 200K lines of Fortran95/2003/2008 code long.

All experiments reported in this section were obtained on either FERMI, located in Bologna (Italy) at CINECA, or JUQUEEN, located in Jülich (Germany) at the Jülich Supercomputing Center (JSC). They belong to the next generation of IBM Blue Gene family of supercomputers, the so-called BG/Q supercomputers. In particular, FERMI is configured as a 10-rack system, featuring a total of 10,240 compute nodes, and JUQUEEN as a 28-rack one with a total of 28,672 compute nodes. These nodes are interconnected by an extremely low-latency five-dimensional (5D) torus interconnection network. Each compute node is equipped with a 16-core, 4-way hardware threaded core, IBM Power PC A2 processor, and 16 GBytes of SDRAM-DDR3 memory (i.e., 1GByte/core), and runs a lightweight proprietary CNK Linux kernel. The codes were compiled using IBM XLF Fortran compilers for BG/Q (v14.1) with recommended optimization flags. The customized MPICH2 library available on these systems was used for message-passing. The codes were linked against the BLAS/LAPACK available on the single-threaded IBM ESSL library for BG/Q (v5.1), and HSL_MA87 (v2.1.1).

5.2. 3D Laplacian results. In this section we study the weak scalability of the MLBDDC-PCG solver codes in FEMPAR for the solution of the 3D Laplacian problem on the unit cube $\Omega=[0,1] \times[0,1] \times$ $[0,1]$, with a constant forcing term $f=1$, and homogeneous Dirichlet boundary conditions on the whole boundary $\partial \Omega$. We consider a global conforming uniform mesh (partition) of $\Omega$ into hexahedra and a trilinear FE discretization (i.e., Q1 FEs). This 3D mesh is partitioned into a cubic grid of $p_{1}{ }^{\frac{1}{3}} \times p_{1}{ }^{\frac{1}{3}} \times p_{1}{ }^{\frac{1}{3}}$ cubic subdomains, with $p_{1}$ being the total number of first level subdomains. The first level subdomain size is $m_{1} 1^{\frac{1}{3}} \times m_{1} \frac{1}{3} \times m_{1}{ }^{\frac{1}{3}} \mathrm{FEs}$. The size of the global FE mesh is therefore equal to $\left(m_{1} p_{1}\right)^{\frac{1}{3}} \times\left(m_{1} p_{1}\right)^{\frac{1}{3}} \times$ $\left(m_{1} p_{1}\right)^{\frac{1}{3}}$.

5.2.1. Sensitivity of preconditioner robustness to subdomain aggregation. In this section we study, for a three-level BDDC preconditioner, the impact that the coarsening ratio $m_{2}$ governing aggregation (of first level subdomains into second level ones) has on preconditioner robustness. This will be measured as the number of iterations that the preconditioned iterative solver takes to meet the convergence criteria.

In Figs. 5.1 and 5.2 we plot the number of PCG iterations ( $y$-axis) as a function of $p_{1}$ ( $x$-axis) for the three-level BDDC(ce) and BDDC(cef) preconditioners, respectively. We set the initial solution vector guess $x^{0}=0$ (see line 3 of Alg. 1), and the PCG iteration was stopped whenever the residual $r_{1}^{k}$ at a given iteration $k$ satisfied $\left\|r_{1}^{k}\right\|_{2} \leq 10^{-6}\left\|r_{1}^{0}\right\|_{2}$. This set-up also applies to the rest of experiments in this paper. We kept fixed $p_{3}=1$, while the number of subdomains in the first and second levels were scaled proportionally as $p_{1}=m_{2} p_{2}$, and $p_{2}=k^{3}$, respectively, with $k=2,3,4, \ldots$, subject to the constraint that $p_{1}+p_{2}+p_{3}$ can at most be 458,762 , i.e., the number of cores available on JUQUEEN. We considered several values $m_{1}=10^{3}, 20^{3}, 30^{3}$ and $40^{3}$ for the size of first level subdomains, and for each value of $m_{1}$, the number of first level subdomains per second level subdomain was varied as $m_{2}=4^{3}, 8^{3}, 12^{3}, 14^{3}$ and $16^{3}$ in order to determine the sensitivity of the preconditioner robustness to $m_{2}$. The $y$-axis of the plots in Fig. 5.2 was scaled to match the ones in Fig. 5.1 to increase readability.

As can be observed from Figs. 5.1 and 5.2, and expected from condition number bounds (see Theorem 2.1), the profile of all plots is such that an asymptotically constant number of PCG iterations is (or will finally be) reached beyond some value of $p_{1}$. The sensitivity of the robustness of the three-level BDDC(ce) preconditioner with respect to $m_{2}$ can be observed in Fig. 5.1; a much lower impact can be observed in Fig. 5.2 for the three-level BDDC(cef) preconditioner. The general trend for "small enough" 

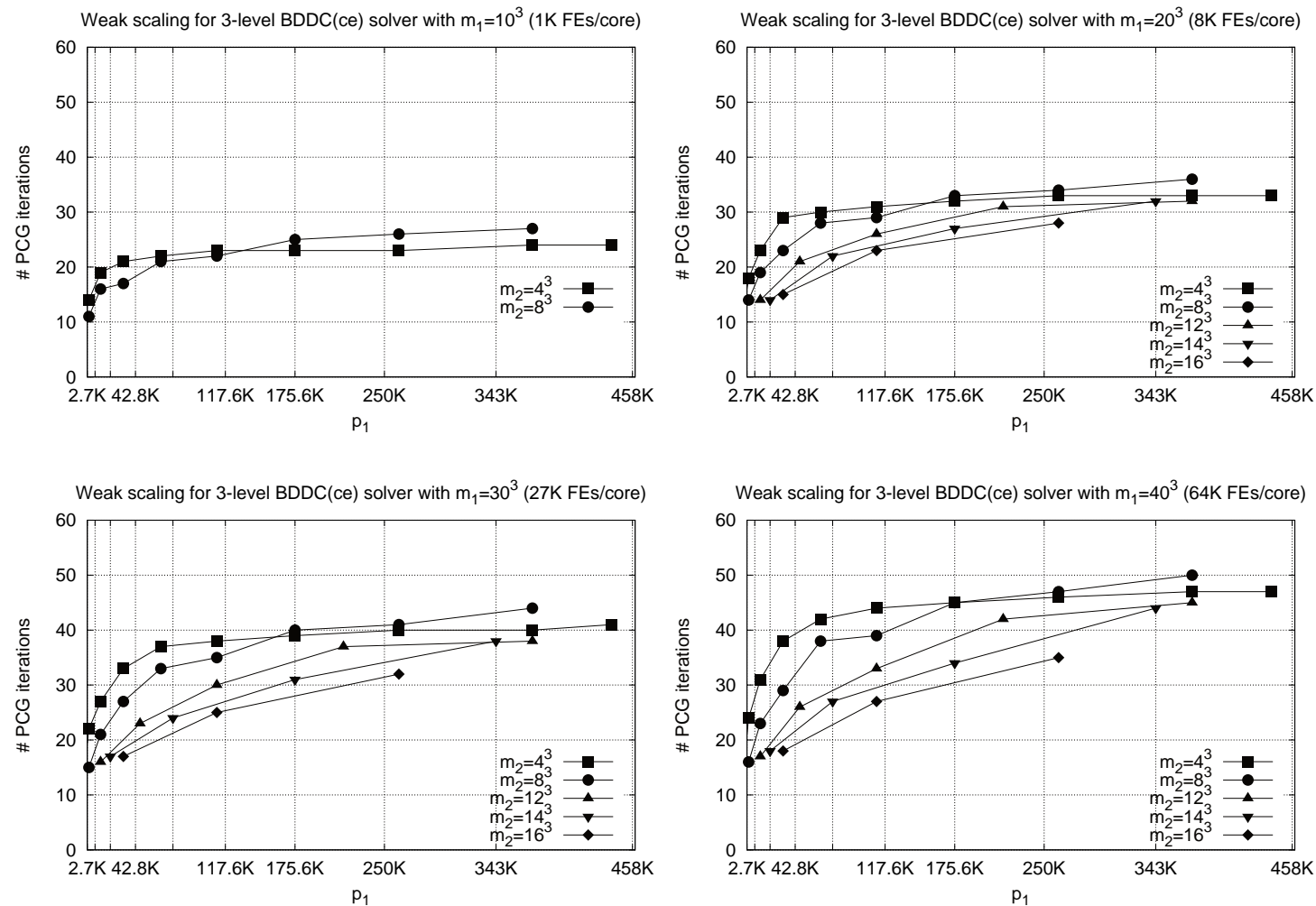

FIG. 5.1. Sensitivity of the number of three-level BDDC(ce)-PCG solver iterations to $m_{2}=4^{3}, 8^{3}, 12^{3}, 14^{3}$ and $16^{3}$. From top to bottom, and left to right: $m_{1}=10^{3}, 20^{3}, 30^{3}$ and $40^{3}$, respectively.

values of $p_{1}$ is that, for fixed $p_{1}$ and $m_{1}$, the larger the value of $m_{2}$, the smaller the number of PCG iterations. For example, for $p_{1}=110 \mathrm{~K}$, and $m_{1}=40^{3}$, the number of PCG iterations is 44, 39, 33, and 27, for $m_{2}=4^{3}, 8^{3}, 12^{3}$ and $16^{3}$, respectively (see Fig. 5.1). This counter-intuitive observation (with respect to condition number bounds) can be explained by the fact that the two-level BDDC preconditioner robustness for the (coarse-grid) linear system gets favoured by very small second level subdomain grids for small $p_{1}$ and large $m_{2}$.

However, $m_{2}$ also impacts how fast the asymptotic regime is reached, and the asymptotically constant number of PCG iterations which is reached. In particular, for fixed $m_{1}$, the larger the value of $m_{2}$, the slower it takes to reach asymptotically constant number of iterations, but the higher the asymptotic number of iterations is. This justifies the crossover which is observed among the plots corresponding to some combinations of $m_{2}$ (e.g., for $m_{2}=4^{3}$ and $m_{2}=8^{3}$ in the top left corner of Fig. 5.1). In any case, the balance reached is such that this crossover is not achieved in most of the cases, or it is only achieved for "large" $p_{1}$, within the range of core counts of interest. This may in fact have a positive (unintended) effect on the performance of the strategy suggested in Sect. 3.3, i.e., maximize $m_{2}$ while keeping the blue regions below the green ones in Table 3.1.

Finally, by comparing the plots in Figs. 5.1 with the ones in 5.2 it can be observed that the three-level $\operatorname{BDDC}$ (cef), apart from being less sensitive to the choice of $m_{2}$, is also more robust than the three-level BDDC(ce) preconditioner, as it takes less iterations to converge, specially for large values of $m_{1}$. This enhanced robustness becomes at the price of heavier second and third level coarse-grid problems. In Sect. 5.2.3, we will evaluate to what extent this increase can still be absorbed by means of inter-level overlapping.

5.2.2. Scalability under low loads on FERMI. In this section we study the weak scalability of the MLBDDC-PCG solver codes on FERMI with "small" first level subdomain sizes, in particular, of $m_{1}=10^{3}(1 \mathrm{~K})$ and $15^{3}(3.4 \mathrm{~K})$ FEs. For these values of $m_{1}$, memory consumption per core for MPI 

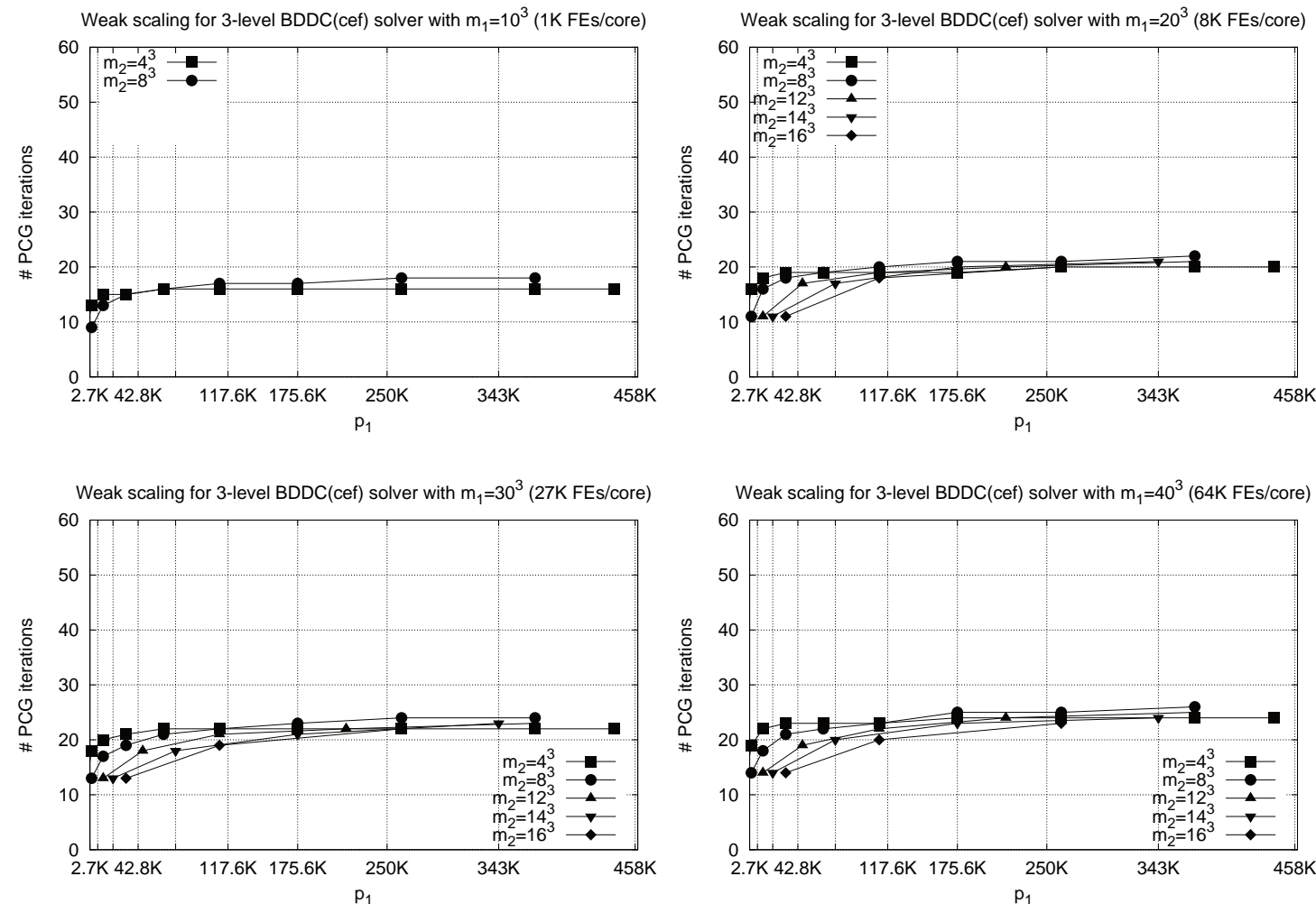

FIG. 5.2. Sensitivity of the number of three-level BDDC(cef)- $P C G$ solver iterations to $m_{2}=4^{3}, 8^{3}, 12^{3}, 14^{3}$ and $16^{3}$. From top to bottom, and left to right: $m_{1}=10^{3}, 20^{3}, 30^{3}$ and $40^{3}$, respectively.

tasks at the first level is very moderate compared to the 1GB available per core on FERMI, in particular of 19.7 and 29.9 MB, respectively. On the other hand, the time spent in the green areas of Table 3.1 is also very moderate, posing a challenge to the full effectiveness of the approach proposed in this paper (see Sect. 3.3).

Figs. 5.3(a) and (b) show, up to 64K FERMI cores, the weak scalability for the total computation time and number of PCG iterations, respectively, for the three-level (solid lines) and four-level (dashed lines) BDDC(ce) (left-hand side) and BDDC(cef) (right-hand side) solvers, with $m_{1}=10^{3}$ (colored in black), and $m_{1}=15^{3}$ (colored in blue). We consider two different set-ups for the three-level BDDC preconditioner, corresponding to $m_{2}=4^{3}$, and $m_{2}=6^{3}$, respectively, while a single one for the four-level BDDC, in particular, $m_{2}=3^{3}$, and $m_{3}=3^{3}$. For the three-level BDDC, we kept fixed $p_{3}=1$, while the number of subdomains in the first and second levels were scaled proportionally as $p_{1}=m_{2} p_{2}$, and $p_{2}=k^{3}$, respectively, with $k=2,3,4, \ldots$. For example, the five points of the plots corresponding to $m_{2}=6^{3}$ (see, e.g, curve with unfilled squares in the right-hand side of Fig. 5.3(b)), are obtained with $k=2,3,4,5$ and 6 , respectively. For the four-level BDDC, the number of first, second, and third level subdomains were scaled proportionally as $p_{1}=m_{2} p_{2}, p_{2}=m_{3} p_{3}, p_{3}=k^{3}$, with $k=2,3,4$, so that we only have three points for the four-level BDDC plots.

The results of Fig. 5.3 clearly reveal three different scenarios for the balance achieved among the time spent in the colored regions in Table 3.1. First, for the three-level BDDC with $m_{2}=4^{3}$, a heavy third level is revealed (i.e., too much time spent in the red areas of the table). For example, if we focus on the scalability of the three-level BDDC(ce) solver with $m_{1}=10^{3}$ on the left-hand side of Fig. 5.3(a), we can see that scalability starts (significantly) degrading beyond $p_{1}=4 \mathrm{~K}$ cores; this degradation is even more severe for the three-level BDDC(cef) solver (see right-hand side of the figure), due to a heavier coarsest-grid problem. In order to (try to) get rid of this, one can be more aggressive when aggregating first level subdomains into second level ones, that is, to consider a larger value of $m_{2}=6^{3}$, leading to a second interesting scenario in Fig. 5.3. With such choice of $m_{2}$, we reduce the size of the coarsest- 

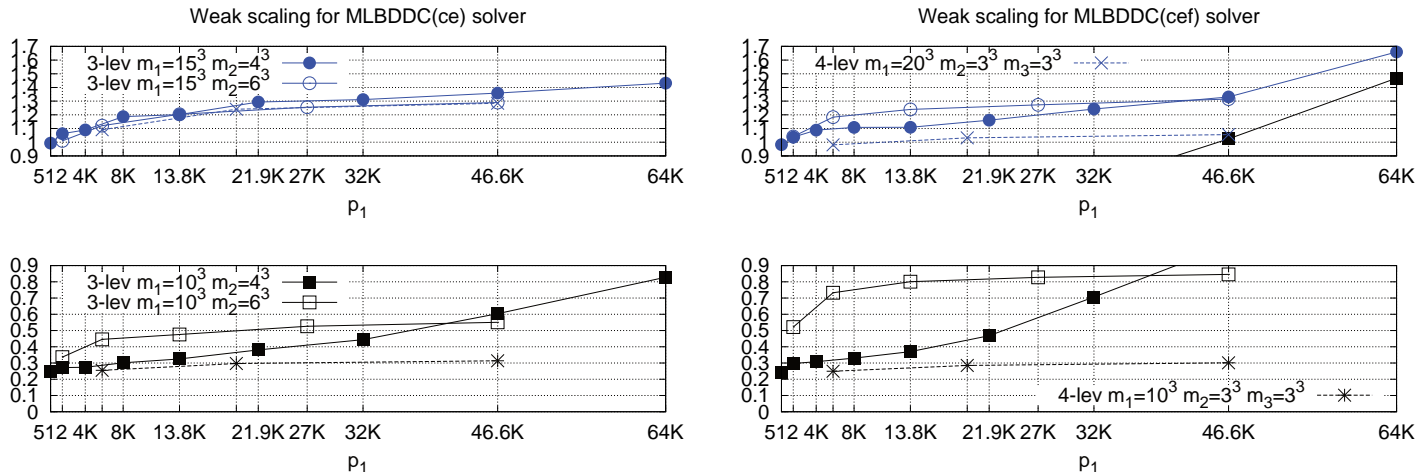

(a) Total computation time (secs.).
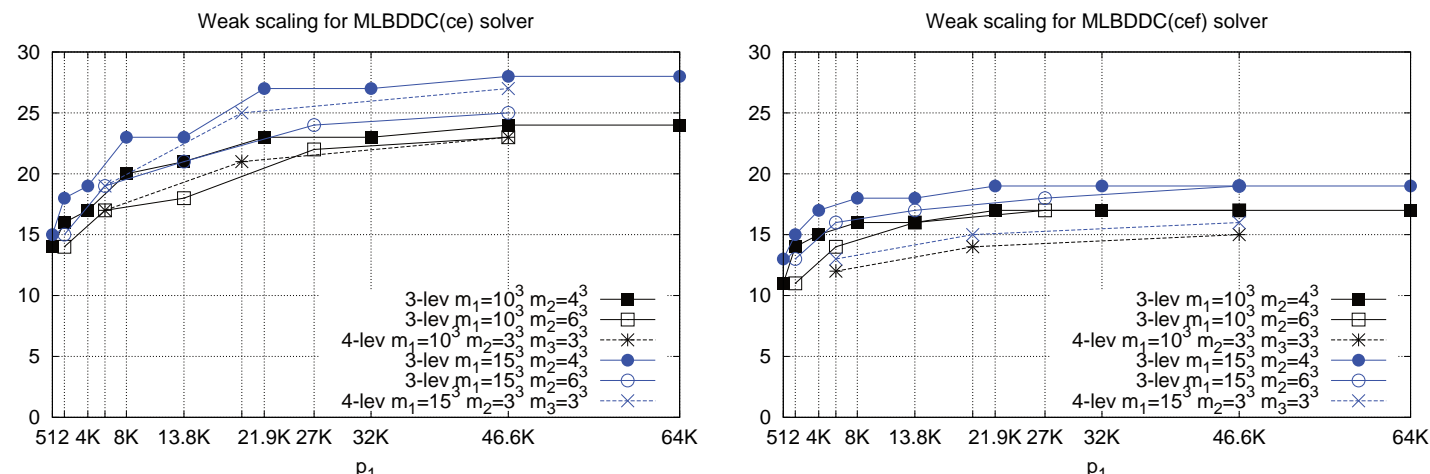

(b) Number of PCG iterations.

FIG. 5.3. Weak scalability for the total computation time (a) and number of PCG iterations (b) of the MLBDDC(ce) (left) and $M L B D D C\left(\right.$ cef) (right) solvers in the solution of the $3 D$ Laplacian PDE with $m_{1}=10^{3}$ and $15^{3}$ FEs on FERMI.

grid problem (and therefore the time spent in the red areas of Table 3.1), but we increase the size of second level subdomains. In particular, as can be observed from Fig. 5.3(a) with $m_{1}=10^{3}$, up to an extent that now a heavy second level is revealed. This is confirmed by the (initially) higher computation times of the three-level BDDC solver with $m_{2}=6^{3}$ compared to those of $m_{2}=4^{3}$. We stress that, although in this scenario an asymptotically constant computation time is reached (up to $64 \mathrm{~K}$ cores), a lot of computational resources are wasted (and therefore parallel and energy efficiency), as first-level cores have to wait (for second-level ones) on communication stages among these two levels. The final (desirable) scenario is the one corresponding to the four-level BDDC method, which reveals a balance such that full effectiveness is achieved. For small $p_{1}$, computational times of the four-level BDDC method are comparable to those of the three-level BDDC method with $m_{2}=4^{3}$ (actually smaller for the former due to an smaller $m_{2}=3^{3}$ ), as in both cases first level duties can mask coarser-grid duties (i.e., the green areas in Table 3.1 dominate). As we scale $p_{1}$, the four-level BDDC can still maintain the desired balance (within the range of core counts studied), as it cuts down the time spent in red areas by the introduction of an additional level in the hierarchy.

5.2.3. Scalability under medium and high loads on JUQUEEN. In this section we study the weak scalability of the MLBDDC-PCG solver codes up to the full JUQUEEN 28-rack IBM BG/Q system, with larger first level subdomain sizes than those considered in Sect. 5.2.2, in particular, of $m_{1}=20^{3}(8 \mathrm{~K}), 25^{3}(15.6 \mathrm{~K}), 30^{3}(27 \mathrm{~K})$, and $40^{3}(64 \mathrm{~K})$ FEs. Memory consumption per first level MPI task was in this case of $80,146,233$, and $651 \mathrm{MB}$, respectively.

Figs. 5.4(a) and (b) show, up to the full JUQUEEN system (i.e., 458,762 cores), the weak scalability for the total computation time and number of PCG iterations, respectively, for the three-level (solid lines) and four-level (dashed lines) BDDC(ce) (left-hand side) and BDDC(cef) (right-hand side) solvers, with $m_{1}=20^{3}$ (colored in black), $25^{3}$ (colored in blue), $30^{3}$ (colored in red), and $40^{3}$ (colored in green) 
FEs. The results for the four-level BDDC are only reported as a reference for the first two values of $m_{1}$. We consider $m_{2}=7^{3}$ for the three-level BDDC preconditioner, and $m_{2}=4^{3}$, and $m_{3}=3^{3}$ for the four-level BDDC one. The number of subdomains in each level were proportionally scaled as in Sect. 5.2.2, with $k$ being at most 6 and 11, respectively.
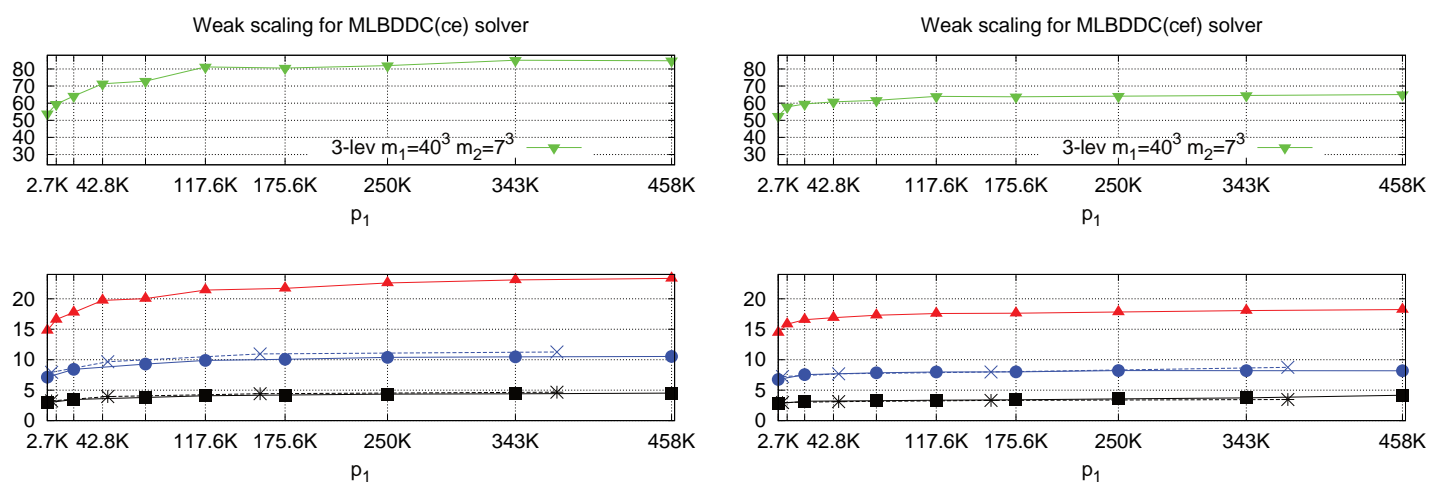

(a) Total computation time (secs.).
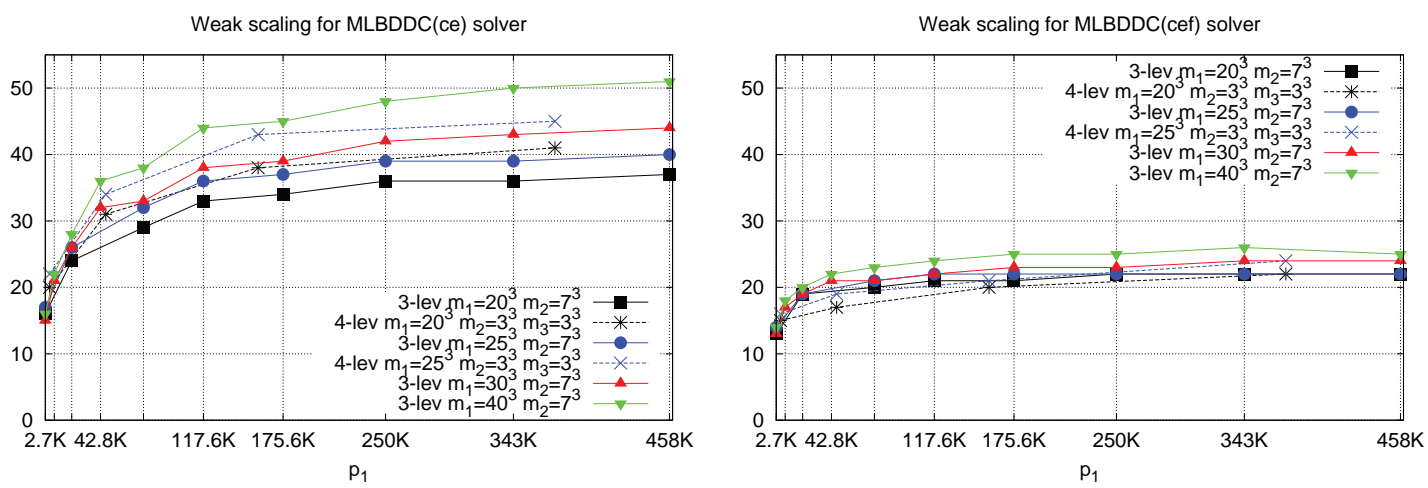

(b) Number of PCG iterations.

FIG. 5.4. Weak scalability for the total computation time (a) and number of PCG iterations (b) of the MLBDDC(ce) (left) and $M L B D D C$ (cef) (right) solvers in the solution of the $3 D$ Laplacian PDE with $m_{1}=20^{3}, 25^{3}, 30^{3}$ and $40^{3}$ FEs on JUQUEEN.

Remarkable scalability can be observed in Fig. 5.4(a) for the three-level BDDC-PCG solver up to full 28-rack IBM BG/Q system, in a practical demonstration of the tremendous potential of the algorithms and codes subject of study. For all values of $m_{1}$ considered, the time spent in green regions of Table 3.1 is "sufficiently large" up to an extent that full effectiveness can already be obtained with a three-level method in the whole range of core counts of interest. In other words, a balance can be struck such that first level duties completely absorb the latency associated to coarser-grid levels. This is fulfilled for both the BDDC(ce), and BDDC(cef) preconditioner, despite the additional work incurred in coarser-grid levels by the introduction of face constraints in the latter BDDC space. This can be readily observed in Fig. 5.4 by the fact that the increased robustness of the BDDC(cef) (i.e., less number of PCG iterations in Fig. 5.4(b)) immediately translates in lower computation times (compare the plots in the left and right-hand side of Fig. 5.4(a)). Another observation than confirm all these evidences is that the computational times of the four-level BDDC preconditioner are very close to that of the threelevel BDDC preconditioner (and therefore three-level BDDC method suffices to keep under control the growth of the time spent in the coarsest-grid level).

5.2.4. Rising the challenge: extra concurrency via overdecomposition. In this section we study the weak scalability of the MLBDDC-PCG solver codes under a partition of the problem into more subdomains than physical cores involved in the parallel computation (i.e., under an overdecomposition of the problem at hand). The cores of the IBM BG/Q supercomputer require that at least two instructions 
are issued per cycle from different hardware threads in order to fully fill their instruction pipeline [17] (and therefore achieve peak flop performance). Therefore, using more MPI tasks per physical core it might be possible to improve the aggregated efficiency of the parallel computation. ${ }^{\dagger}$ We in particular explore in this section 4 MPI tasks/core. (We also performed experiments with 2 MPI tasks/core, although the results with 4 MPI tasks/core confirm a higher profit from the hardware threads in terms of aggregated efficiency.) However, the usage of this technique significantly challenges scalability of the algorithm/code/hardware combination. In particular, 4 MPI tasks/core implies a very moderate amount of memory of 256MB/MPI task, and a 4-fold increase in the coarse-grid problem size to be solved at each level of the hierarchy. This challenge is, however, aligned to current/and future HPC trends of having much more concurrency and less memory per core. Besides, each physical core becomes responsible for the computation and communication of four different subdomains. In order to cope with a smaller load per core, and larger coarse-grid problems, we had to consider a four-level BDDC preconditioner to cope for the growth of time spent in the coarsest-grid level.

Figs. 5.5(a) and (b) report the weak scalability for the total computation time and number of PCG iterations, respectively, for the four-level BDDC(ce) and BDDC(cef) solvers, with $m_{1}=10^{3}$ (colored in black), $20^{3}$ (colored in blue), and $25^{3}$ (colored in red) FEs. We considered $m_{2}=4^{3}$, and $m_{3}=3^{3}$, scaling the number of first, second, and third level MPI tasks proportionally as $p_{1}=m_{2} p_{2}, p_{2}=m_{3} p_{3}$, $p_{3}=k^{3}$, with $k=2,3,4, \ldots, 10$. The resulting number of tasks were mapped to 4 time less cores (i.e., $4 \mathrm{MPI}$ tasks/core). Therefore, for the largest value of $k$, we mapped 1.73M first level subdomains on 448.3K cores.
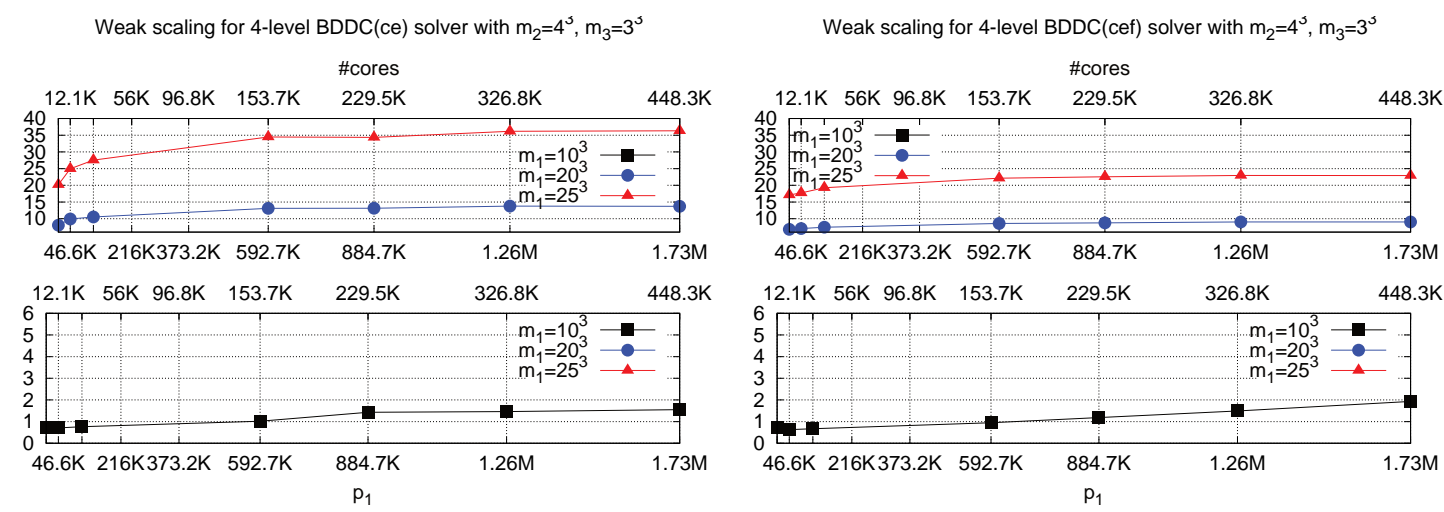

(a) Total computation time (secs.).

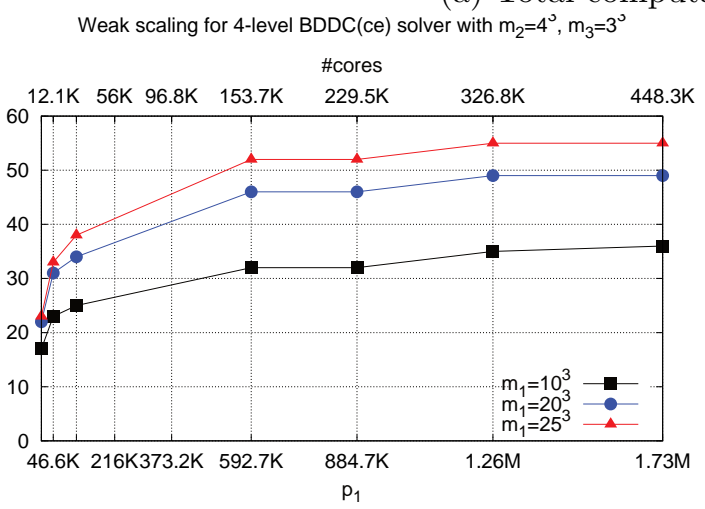
Weak scaling for 4-level BDDC(cef) solver with $\mathrm{m}_{2}=4^{\circ}, \mathrm{m}_{3}=3^{\circ}$

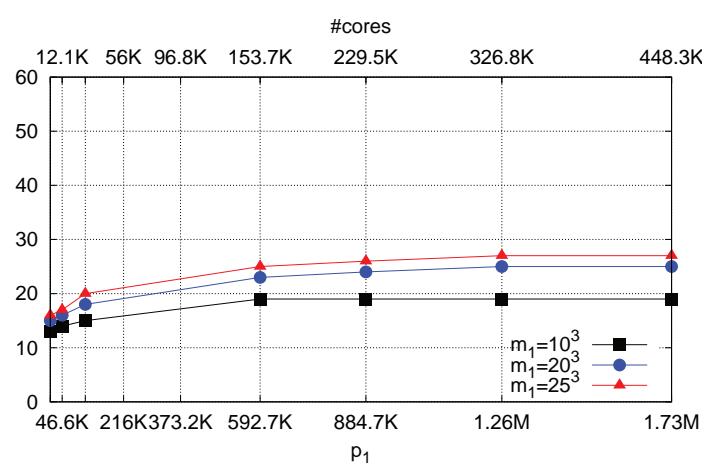

(b) Number of PCG iterations.

FIG. 5.5. Weak scalability for the total computation time (a) and number of PCG iterations (b) of the four-level $B D D C(c e)$ (left) and $B D D C\left(\right.$ cef) (right) solvers in the solution of the $3 D$ Laplacian PDE with $m_{1}=10^{3}, 20^{3}$ and $25^{3}$ FEs on JUQUEEN with 4 MPI tasks/core.

${ }^{\dagger}$ Due to restrictions inherent to the IBM BG/Q supercomputer software/hardware stack, this can be done at most up to 4 MPI tasks/physical core (i.e., 4 subdomains handled by each physical core). 
As can be observed in Fig. 5.5, still remarkable scalability is achieved with our approach despite the 4-fold increase in the number of subdomains. In particular, full absorption of coarse-grid duties is achieved by means of interlevel-overlapping for all combinations of preconditioner and $m_{1}$, except for the four-level BDDC(cef) and $m_{1}=10^{3}$, where a mild degradation of scalability is achieved beyond $p_{1}=592.7 \mathrm{~K}$ subdomains. We stress that we did not actually fine-tuned the values of $m_{2}$ and $m_{3}$, nor the number of levels for this experiment. A larger value of $m_{3}$ (e.g. $\left.4^{3}\right)$, or a further level, could improve the situation for this particular case.

Apart from confirming remarkable scalability, we compared, on smaller test cases, the computation times of the codes using 16 and 64 MPI tasks/node (with the same number of MPI tasks/level in both cases), confirming an approximately $50 \%$ save in aggregated efficiency by the exploitation of hardware multi-threading (i.e., the computation time with 64 MPI tasks/node was approximately twice as much the one with 16 MPI tasks/node).

5.3. 3D linear elasticity results. In this section we evaluate the weak scalability of the MLBDDC-PCG codes when applied to a vector-valued problem, namely the Q1 FE approximation of the (compressible) 3D linear elasticity PDE (with first and second Lamé parameters equal to 1 and $\frac{1}{10}$, respectively) on a unit cube $\Omega=[0,1] \times[0,1] \times[0,1]$, a constant forcing term $f=1$, and homogeneous Dirichlet boundary conditions on the whole boundary. The same experiment set-up to that selected for the three-level BDDC preconditioner in Sect. 5.2.3 is considered here, except for the size of first level subdomains, which we now set-up as $m_{1}=15^{3}(3.4 \mathrm{~K}), 20^{3}(8 \mathrm{~K})$, and $25^{3}(15.6 \mathrm{~K})$ FEs. The largest subdomain size in this set is much smaller than the largest one considered for the 3D Laplacian problem in Sect. 5.2 .3 (i.e., $m_{1}=40^{3}$ ). The linear elasticity problem is a vector-valued problem with 3 unknowns per FE mesh node. This implies that, for a given FE mesh, the size of the discrete operator is 3 times larger than that of the Laplacian problem, and has 9 times more nonzero entries. Indeed, with $m_{1}=25^{3}$ the memory consumption per first level MPI task is $713 \mathrm{MB}$, compared to $146 \mathrm{MB}$ for the same value of $m_{1}$ in the case of the 3D Laplacian problem.

Fig. 5.6(a) and (b) report, up to the full 28-rack IBM BG/Q system, the weak scalability for the total computation time and number of PCG iterations, respectively, for the three-level BDDC(ce) and BDDC(cef) solvers, with $m_{1}=10^{3}$ (colored in black), $20^{3}$ (colored in blue), and $25^{3}$ (colored in red) FEs.

As evidenced in Fig. 5.6, the approach pursued in this paper is also able to obtain remarkable scalability even for a much more computationally intensive problem such as the linear elasticity problem. Provided a "sufficiently large" $m_{1}$, i.e., $20^{3}$ and $25^{3}$ FEs for BDDC(ce) and BDDC(cef), respectively, a MLBDDC hierarchy equipped with 3 levels is already sufficient to fully overlap coarser-grid duties in the full range of cores available on the JUQUEEN supercomputer. For smaller values of $m_{1}$, some (mild) degradation of scalability is observed beyond some point, e.g., for $15^{3}$, beyond $p_{1}=175.6 \mathrm{~K}$ first level subdomains, which would require an additional level to keep perfect scalability.

6. Conclusions and future work. In this article, we have presented a highly scalable parallel implementation of exact MLBDDC methods, i.e., based on sparse direct solvers for the local (and coarsest) problems. The proposed implementation is based on recursion and communicator-awareness, in order to strategically deploy MPI tasks with duties at only one level and overlap interlevel tasks, based on the fact that fine and coarse corrections at every level of the MLBBDC method can be computed in parallel. The result is a MPMD and bulk-asynchronous implementation with reduced synchronization among cores, which overlaps communications/computations of the different levels. Due to the recursive implementation, it can be used for an arbitrary number of levels.

This implementation leads to close to perfect weak scalability results as far as (embarrassingly parallel) level-1 duties can mask tasks at higher levels. We have provided a model that helps us to choose effective coarsening ratios among cores and indicates when the overlapped strategy will start loosing effectiveness. In any case, the main motivation of this work is not only to attain excellent weak scalability but also to reduce drastically the aggregated idling, via the interlevel-overlapped implementation. It turns out in improved parallel efficiency, energy awareness, and reduced time-to-solution.

A detailed scalability analysis has been carried out for the proposed implementation of the algorithms, reaching the whole JUQUEEN Blue Gene/Q, up to 458,752 cores and 1.8 million MPI tasks (subdomains), for both Laplacian and linear elasticity problems. These are the largest scale problems 

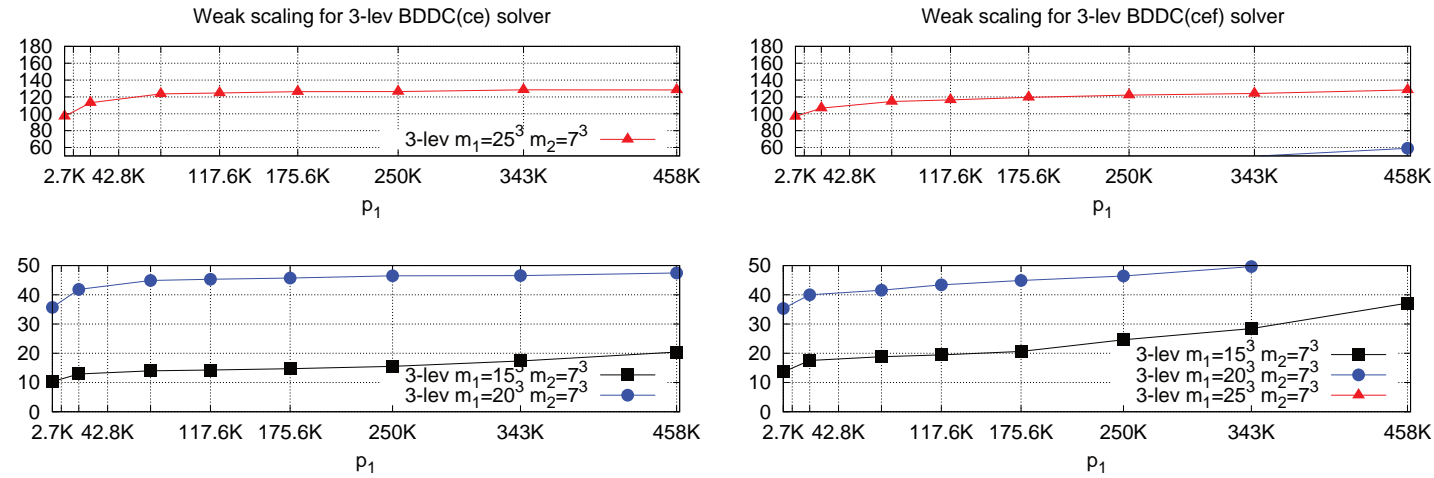

(a)
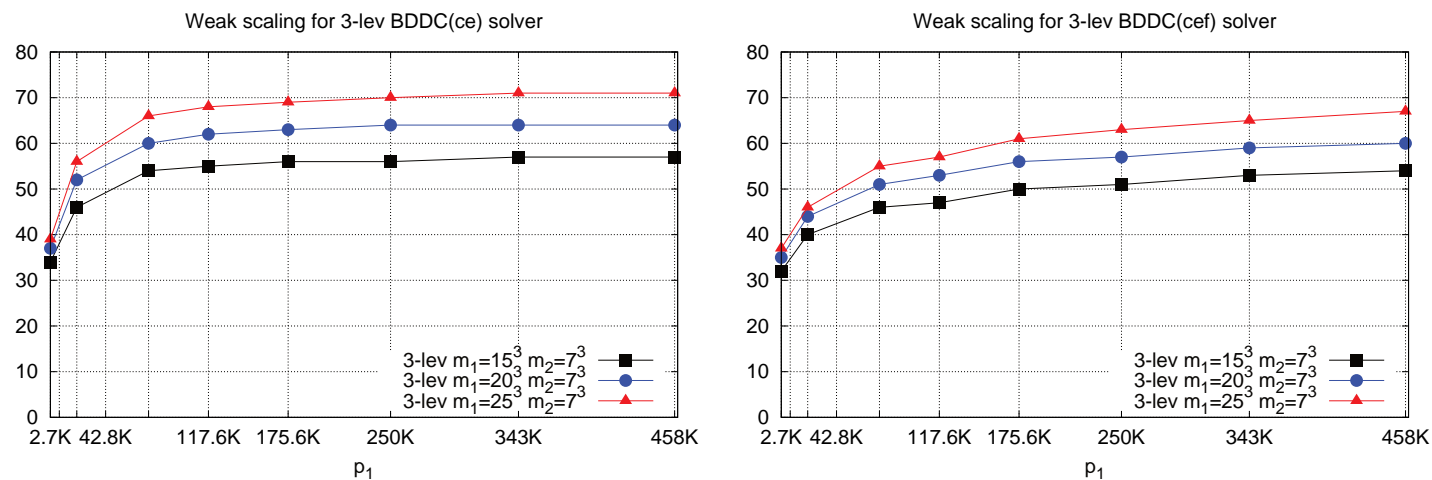

(b)

FIG. 5.6. Weak scalability for the total computation time (a) and number of PCG iterations (b) of the three-level $B D D C(c e)$ (left) and $B D D C\left(\right.$ cef) (right) solvers in the solution of the $3 D$ linear elasticity PDE with $m_{1}=10^{3}, 20^{3}$ and $25^{3}$ FEs on JUQUEEN.

reported so far for exact DD preconditioners. Both three-level and four-level algorithms have been explored. In order to stress the proposed implementation, (1) the coarsest-level problem has been solved in only one processor, i.e., no parallelization has been exploited at the last level, and (2) we have considered overdecomposition, i.e., to use a partition of the problem into more subdomains than physical cores involved in the parallel computation, reaching up to $1.8 \mathrm{M}$ subdomains. The results show a close to perfect weak scalability for low to moderate local problem sizes, i.e., infra-utilizing the memory resources per core. The use of more than four levels or a distributed computation of the coarsest problem can be of interest with the thousand-fold increase in the number of cores expected in the near future.

Future work will include the extension of the framework to Krylov-cycling MLBDDC methods, in order to increase preconditioner robustness and keep constant the number of iterations as we go to more levels, and the development of inexact MLBDDC (hybrid AMG-BDDC) algorithms and implementations. We finally note that this implementation approach can also be applicable to other preconditioners, like the BPX (additive MG) algorithm [9], in order to improve parallel efficiency, time-to-solution, and weak scalability.

\section{References.}

[1] Report on the workshop on extreme-scale solvers: Transition to future architectures, U.S. Department of Energy, 2012.

[2] P.R. Amestoy, I.S. Duff, and J.-Y. L'Excellent, Multifrontal parallel distributed symmetric and unsymmetric solvers, Computer Methods in Applied Mechanics and Engineering 184 (2000), no. 24, 501-520.

[3] S. Badia, A. F. Martín, and J. Principe, Enhanced balancing Neumann-Neumann preconditioning in computational fluid and solid mechanics, International Journal for Numerical Methods in Engineering 96 (2013), no. 4, $203-230$.

[4] __ Implementation and scalability analysis of balancing domain decomposition methods, Archives of Computational Methods in Engineering 20 (2013), no. 3, 239-262. 
[5] _ A A highly scalable parallel implementation of balancing domain decomposition by constraints, SIAM Journal on Scientific Computing (2014), C190-C218.

[6] _ On an overlapped coarse/fine implementation of balancing domain decomposition with inexact solvers, Submitted (2014).

[7] A. H. Baker, T. Gamblin, M. Schulz, and U. M. Yang, Challenges of scaling algebraic multigrid across modern multicore architectures, Parallel distributed processing symposium (IPDPS), 2011 IEEE international, 2011, pp. 275 $-286$.

[8] S. Balay, J. Brown, K. Buschelman, W. D. Gropp, D. Kaushik, M. G. Knepley, L. C. McInnes, B. F. Smith, and H. Zhang, PETSc Web page, 2012. http://www.mcs.anl.gov/petsc.

[9] J. H. Bramble, J. E. Pasciak, and J. Xu, Parallel multilevel preconditioners, Mathematics of Computation 55 (1990), no. $191,1-22$.

[10] S. C. Brenner and R. Scott, The mathematical theory of finite element methods, 3rd edition, Springer, 2010.

[11] D. Brömmel and P. Gibbon, High-Q Club The highest scaling Codes on JUQUEEN, Innovatives Supercomputing in Deutschland 11 (2013), no. 2, 106-107.

[12] T. A. Davis, Direct methods for sparse linear systems, Vol. 2, SIAM, 2006.

[13] C. R. Dohrmann, A preconditioner for substructuring based on constrained energy minimization, SIAM Journal on Scientific Computing 25 (2003), no. 1, 246-258.

[14] _ An approximate BDDC preconditioner, Numerical Linear Algebra with Applications 14 (2007), no. 2, 149168 (en).

[15] C. Farhat, K. Pierson, and M. Lesoinne, The second generation FETI methods and their application to the parallel solution of large-scale linear and geometrically non-linear structural analysis problems, Computer Methods in Applied Mechanics and Engineering 184 (2000), no. 2-4, 333-374.

[16] V. Hapla, D. Horak, and M. Merta, Use of direct solvers in TFETI massively parallel implementation, Applied parallel and scientific computing, 2013, pp. 192-205.

[17] R.A. Haring, M. Ohmacht, T.W. Fox, M.K. Gschwind, D.L. Satterfield, K. Sugavanam, P.W. Coteus, P. Heidelberger, M.A. Blumrich, R.W. Wisniewski, A. Gara, G.L.-T. Chiu, P.A. Boyle, N.H. Chist, and C. Kim, The IBM blue Gene/Q compute chip, IEEE Micro 32 (2012), no. 2, 48-60.

[18] J. Hogg, J. Reid, and J. Scott, Design of a multicore sparse cholesky factorization using DAGs, SIAM Journal on Scientific Computing 32 (2010), no. 6, 3627-3649.

[19] G. Karypis, A software package for partitioning unstructured graphs, partitioning meshes, and computing fill-reducing orderings of sparse matrices. Version 5.1.0, University of Minnesota, Department of Computer Science and Engineering, Minneapolis, MN, 2013. Available at http://glaros.dtc.umn.edu/gkhome/fetch/sw/metis/manual.pdf.

[20] A. Klawonn and O. Rheinbach, Highly scalable parallel domain decomposition methods with an application to biomechanics, ZAMM - Journal of Applied Mathematics and Mechanics 90 (2010), no. 1, 532 (en).

[21] A. Klawonn and O. B. Widlund, A domain decomposition method with lagrange multipliers and inexact solvers for linear elasticity, SIAM Journal on Scientific Computing 22 (2000), no. 4, 1199-1219.

[22] P. T. Lin, J. N. Shadid, M. Sala, R. S. Tuminaro, G. L. Hennigan, and R. J. Hoekstra, Performance of a parallel algebraic multilevel preconditioner for stabilized finite element semiconductor device modeling, Journal on Computational Physics 228 (2009), no. 17, 6250-6267.

[23] J. Mandel, Balancing domain decomposition, Communications in Numerical Methods in Engineering 9 (1993), no. 3, 233-241.

[24] J. Mandel and C. R. Dohrmann, Convergence of a balancing domain decomposition by constraints and energy minimization, Numerical Linear Algebra with Applications 10 (2003), no. 7, 639-659.

[25] J. Mandel, B. Sousedík, and C. Dohrmann, Multispace and multilevel BDDC, Computing 83 (2008), no. 2, 55-85.

[26] O. Rheinbach, Parallel iterative substructuring in structural mechanics, Archives of Computational Methods in Engineering 16 (2009), no. 4, 425-463 (en).

[27] Y. Saad, Iterative methods for sparse linear systems, 2nd ed., SIAM, 2003.

[28] O. Schenk and K. Gärtner, On fast factorization pivoting methods for sparse symmetric indefinite systems, Electronic Transactions on Numerical Analysis 23 (2006), 158-179.

[29] B. Sousedík, J. Šístek, and J. Mandel, Adaptive-multilevel BDDC and its parallel implementation, Computing 95 (2013), no. 12, 1087-1119.

[30] K. Stüben, A review of algebraic multigrid, Journal of Computational and Applied Mathematics 128 (2001), no. 12, $281-309$.

[31] A. Toselli and O. Widlund, Domain decomposition methods - algorithms and theory (R. Bank, R. L. Graham, J. Stoer, R. Varga, and H. Yserentant, eds.), Springer-Verlag, 2005.

[32] X. Tu, Three-level BDDC in three dimensions, SIAM Journal on Scientific Computing 29 (2007), no. 4, 1759-1780.

[33] J. Šístek, M. Čertíková, P. Burda, and J. Novotný, Face-based selection of corners in 3D substructuring, Mathematics and Computers in Simulation 82 (2012), no. 10, 1799-1811. 